\title{
Protoplanetary Disks as (Possibly) Viscous Disks
}

\author{
Roman R. Rafikov ${ }^{1,2}$ \\ ${ }^{1}$ Department of Applied Mathematics and Theoretical Physics, Centre for Mathematical Sciences, \\ University of Cambridge, Wilberforce Road, Cambridge CB3 0WA, UK; rrr@damtp.cam.ac.uk \\ 2 Institute for Advanced Study, 1 Einstein Drive, Princeton, NJ 08540, USA \\ Received 2017 January 20; revised 2017 February 15; accepted 2017 February 20; published 2017 March 15
}

\begin{abstract}
Protoplanetary disks are believed to evolve on megayear timescales in a diffusive (viscous) manner as a result of angular momentum transport driven by internal stresses. Here we use a sample of 26 protoplanetary disks resolved by ALMA with measured (dust-based) masses and stellar accretion rates to derive the dimensionless $\alpha$-viscosity values for individual objects, with the goal of constraining the angular momentum transport mechanism. We find that the inferred values of $\alpha$ do not cluster around a single value, but instead have a broad distribution extending from $10^{-4}$ to 0.04 . Moreover, they correlate with neither the global disk parameters (mass, size, surface density) nor the stellar characteristics (mass, luminosity, radius). However, we do find a strong linear correlation between $\alpha$ and the central mass accretion rate $\dot{M}$. This correlation is unlikely to result from the direct physical effect of $\dot{M}$ on internal stress on global scales. Instead, we suggest that it is caused by the decoupling of stellar $\dot{M}$ from the global disk characteristics in one of the following ways: (1) The behavior (and range) of $\alpha$ is controlled by a yetunidentified parameter (e.g., ionization fraction, magnetic field strength, or geometry), ultimately driving the variation of $\dot{M}$. (2) The central $\dot{M}$ is decoupled from the global accretion rate as a result of an instability, or mass accumulation (or loss in a wind or planetary accretion) in the inner disk. (3) Perhaps the most intriguing possibility is that angular momentum in protoplanetary disks is transported nonviscously, e.g., via magnetohydrodynamic winds or spiral density waves.
\end{abstract}

Key words: accretion, accretion disks - planets and satellites: formation - protoplanetary disks

\section{Introduction}

Protoplanetary disks are thought to persist around their parent stars for a relatively short span of time. Observations present a clear evolutionary picture, in which both the fraction of systems exhibiting IR excess (Hillenbrand 2005) and the mass accretion rate onto the central star (Calvet et al. 2000) decline on megayear timescales. Both observational indicators are thought to be the clear signatures of the presence of the circumstellar disks.

Astrophysical accretion disks are believed to evolve predominantly under the action of their internal stresses (Shakura \& Sunyaev 1973; Lynden-Bell \& Pringle 1974), and protoplanetary disks are no exception to the rule. For a long time evolutionary models of the protoplanetary disks have been developed assuming that the disks spread viscously, losing mass to the central star, while at the same time providing the birth site for planet formation. The characteristic time for the disk evolution in these models is simply the viscous time $t_{\nu}$ at the outer radius of the disk $r_{\text {out }}$,

$$
t_{\nu} \approx r_{\text {out }}^{2} / \nu
$$

Here $\nu$ is the kinematic viscosity, which is conveniently parameterized using the $\alpha$-prescription (Shakura \& Sunyaev 1973)

$$
\nu=\alpha c_{s}^{2} \Omega^{-1},
$$

with $\alpha \lesssim 1$ being constant, and where $c_{s} \equiv(k T / \mu)^{1 / 2}$ (T is the disk temperature) and $\Omega \equiv\left(G M_{\star} / r^{3}\right)^{1 / 2}\left(M_{\star}\right.$ is the mass of the central star) are the local sound speed and Keplerian angular frequency, respectively. Viscous models invariably predict that on long timescales (exceeding the viscous time of the initial, more compact disk, so that $r_{\text {out }}$ grows beyond the initial disk radius) the central mass accretion rate $\dot{M}$ should be related to the total disk mass $M_{d}$ as

$$
\dot{M} \approx M_{d} / t_{\nu}
$$

(up to a constant factor of order unity), with $t_{\nu}$ evaluated at $r_{\text {out }}$; see Equation (1).

The idea of the viscous evolution of the protoplanetary disks, diffusive in character and characterized by Equations (1)-(3), has gained certain observational support. In particular, Hartmann et al. (1998) and Calvet et al. (2000) found that the observed average properties of protoplanetary disks can be explained by their viscous evolution, with efficiency corresponding to $\alpha \approx 10^{-2}$.

The value of the dimensionless parameter $\alpha$ is believed to directly reflect the physics of the mechanism responsible for the angular momentum transport in the disk. In hot and wellionized accretion disks around compact objects transport is generally thought to be mediated by the magnetorotational instability (MRI; Velikhov 1959; Chandrasekhar 1960; Balbus \& Hawley 1991). The situation is less clear in the cold and poorly ionized protoplanetary disks, where the nonideal magnetohydrodynamic (MHD) effects are known to weaken or even suppress the transport driven by the MRI (Turner et al. 2014). Other potential candidates such as gravitoturbulence (Gammie 2001; Rafikov 2015), Rossbywave instability (Lovelace et al. 1999), convective overstability (Latter 2016), vertical shear instability (Urpin \& Brandenburg 1998; Stoll \& Kley 2014), and so on, have been proposed to explain the observed evolution of the protoplanetary disk properties.

On the other hand, recent studies argue that the non-MRIrelated transport mechanisms can hardly be responsible for the observed disk evolution on megayear timescales, because of 
the weakness of the purely hydrodynamic transport mechanisms (Stoll \& Kley 2014; Turner et al. 2014). Partly for that reason, the nondiffusive angular momentum transport mechanisms such as MHD winds (Wardle \& Koenigl 1993; Suzuki \& Inutsuka 2009; Bai \& Stone 2013) and spiral shocks (Rafikov 2002, 2016) have been gaining popularity. The distinctive feature of these mechanisms is that they do not need to obey Equations (1)-(3), thus resulting in a different relation between $\dot{M}$ and $M_{d}$.

The advent of ALMA made possible more precise and focused efforts to understand protoplanetary disk evolution. Recent measurements of the continuum and $\mathrm{CO}$ line emission for a large sample of protoplanetary disks in Lupus by Ansdell et al. (2016) and Miotello et al. (2016b), coupled with the most up-to-date determinations of the mass accretion rate $\dot{M}$ onto their parent stars by Alcalá et al. (2014, 2016), allowed Manara et al. (2016) to identify a correlation between the disk mass $M_{d}$ and the central mass accretion rate. The disk masses were derived using the dust masses inferred from the continuum submillimeter emission assuming fixed gas-to-dust ratio. This correlation has been interpreted by Manara et al. (2016) as providing evidence for the viscous character of the protoplanetary disk evolution, in which the global disk properties directly determine the mass accretion rate at its center.

In this work we focus on a different diagnostics of the viscous disk evolution. Using a sample of protoplanetary disks directly resolved by ALMA, with measured dust and gas masses (Ansdell et al. 2016), as well as the central accretion rates (Alcalá et al. 2014, 2016), we provide a direct determination of the value of the $\alpha$-parameter in individual systems. Given that different mechanisms of the angular momentum transport in disks predict different values of $\alpha$, our effort can provide direct information on the physical nature of the internal stresses driving the disk evolution. Unlike other studies (Hartmann et al. 1998; Jones et al. 2012), in this work we (1) utilize information about the individual disk sizes provided by ALMA and (2) do not use information on the ages of the parent stars, which are known to be very uncertain.

Our work is organized as follows. We describe our methodology for inferring $\alpha$ in Section 2 and our observational sample in Section 2.1. Our results, including correlations of $\alpha$ with different characteristics of the observed systems, can be found in Section 3. We provide extensive discussion of our findings in Section 4 and summarize the results in Section 5.

\section{Methodology}

In our analysis we will assume that, as a result of expansion driven by internal stresses, the present-day sizes of the protoplanetary disks in Lupus exceed their initial radii, set at the mass infall phase. Then the disk can be approximately considered as evolving in a self-similar fashion, and Equations (1)-(3) should apply. Their combination yields

$$
\alpha \approx \frac{\dot{M}}{M_{d}} \frac{\mu}{k T} \Omega r^{2},
$$

where $\Omega, r$, and $T$ are evaluated at the outer disk radius $r_{\text {out }}$, and $M_{d}$ is the disk mass enclosed within $r_{\text {out }}$.

In Equation (4) the values of $r_{\text {out }}, M_{d}, \dot{M}$, and $M_{\star}$ come directly from observations. However, to obtain $\alpha$, we still need to make assumptions about the disk temperature $T\left(r_{\text {out }}\right)$. We try three different thermodynamic prescriptions in this work.
First, we simply assume that

$$
T\left(r_{\text {out }}\right)=20 \mathrm{~K} \text {, }
$$

for all disks in our sample. This prescription is the same as that used by Ansdell et al. (2016) for deriving the dust masses $M_{\text {dust }}$ of the disks from their continuum submillimeter fluxes, providing certain internal consistency.

Second, we take the $T\left(r_{\text {out }}\right)$ to correspond to the temperature of optically thin dust, directly illuminated by the central star, in which case

$$
T\left(r_{\text {out }}\right)=\left(\frac{L_{\star}}{16 \pi \sigma r_{\text {out }}^{2}}\right)^{1 / 4} .
$$

This expression neglects the difference between the emission and absorption efficiencies of the grains. Stellar luminosity $L_{\star}$ is known to us from observations.

Finally, we also use a prescription for the optically thick, externally irradiated passive protoplanetary disks, motivated by Chiang \& Goldreich (1997), that reads

$$
T\left(r_{\text {out }}\right)=120 \mathrm{~K}\left(\frac{L_{\star}}{L_{\odot}}\right)^{2 / 7}\left(\frac{M_{\odot}}{M_{\star}}\right)^{1 / 7}\left(\frac{\mathrm{au}}{r_{\text {out }}}\right)^{3 / 7} .
$$

This prescription predicts $T\left(r_{\text {out }}\right)$ lower than in the case given by Equation (6).

We determine the full disk masses using the dust masses $M_{\text {dust }}$ derived from the continuum submillimeter fluxes (Ansdell et al. 2016). To convert $M_{\text {dust }}$ to the full disk mass $M_{d}$, we use a uniform gas-to-dust mass ratio $\chi=100$. In certain cases (Section 3.2) we also use the information on the gas masses coming from the ${ }^{13} \mathrm{CO}$ and $\mathrm{C}^{18} \mathrm{O}$ line measurements by ALMA. However, it should be kept in mind that the disk masses inferred this way are believed to be systematically underestimated (see our Figure 1(c)), often by more than an order of magnitude, as a result of $\mathrm{CO}$ freezeout on dust grains or sequestration of carbon into large bodies (Ansdell et al. 2016; Miotello et al. 2016a). As a result, it is expected that $M_{d}=\chi M_{\text {dust }}$, which we employ in this work, should provide a better estimate of the disk mass.

\subsection{Observational Sample}

Our approach to determining $\alpha$ via Equation (4) works only for disks that have nontrivial measurements of $\dot{M}, M_{d}$, and $r_{\text {out }}$, as well as of $L_{\star}$ and $M_{\star}$. For this reason, we are interested only in resolved disks with significant detections of both the continuum dust emission by ALMA and the stellar mass accretion rate $\dot{M}$ via spectroscopy.

Ansdell et al. (2016) have carried out an ALMA survey of 89 protoplanetary disks in the Lupus star-forming complex at $\sim 150-200$ pc away from the Sun (age $3 \pm 2$ Myr; Alcalá et al. 2014). They directly resolved many sources and provided initial measurements of the dust and gas masses for about twothirds and one-thirds of their sample, correspondingly. Miotello et al. (2016b) carried out a more sophisticated analysis of this data set based on work of Miotello et al. (2016a), providing more accurate dust and gas mass measurements. At the same time, Alcalá et al. $(2014,2016)$ carried out X-shooter spectroscopy for many of these targets, deriving central mass accretion rates $\dot{M}$ based on the UV excesses.

By examining the samples presented in these studies, we selected 26 objects, which possess resolved disks with well- 

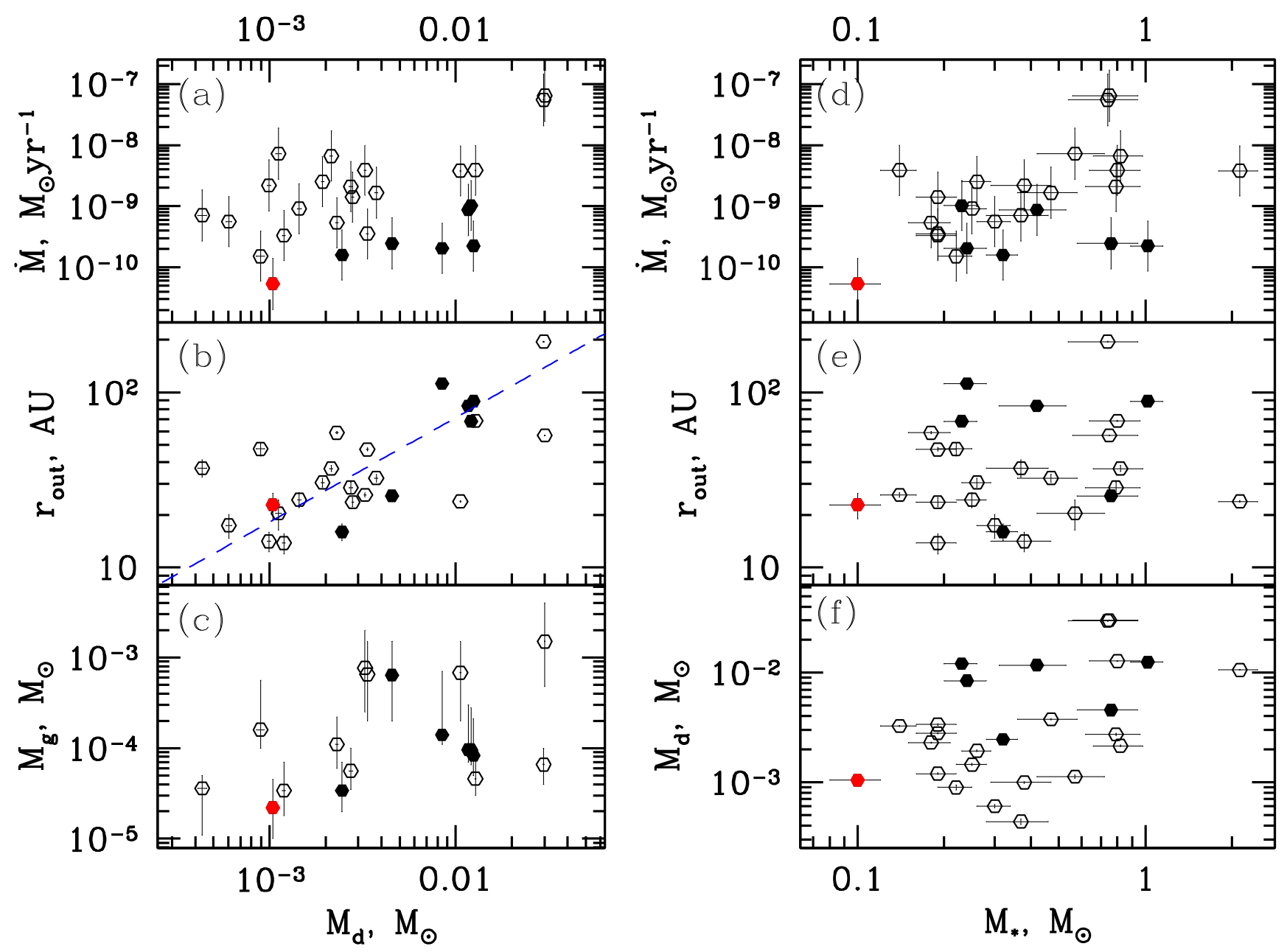

Figure 1. Different characteristics of the systems in the sample of objects used in this work (see Table 1) shown to reveal possible correlations. Quantitative characteristics of the possible correlations can be found in Table 2. Here and in all subsequent plots open (filled) hexagons correspond to objects with the characteristic accretion time $M_{d} / M$ lower (higher) than 10 Myr. Red filled hexagons show object 2MASS J16081497-3857145, which is close to the brown dwarf regime. (a) Central accretion rate $\dot{M}$ vs. disk mass $M_{d}$ inferred from the continuum dust emission. Given the limited size of our sample, we do not find strong evidence for a correlation between $\dot{M}$ and $M_{d}$ in our sample (see Manara et al. 2016). (b) Disk size $r_{\text {out }}$ vs. $M_{d}$, showing a correlation between $r_{\text {out }}$ and $M_{d}$. The linear regression is shown as the blue dashed line (see text for its explicit form). (c) Gas mass inferred from molecular line observations $M_{g}$ (only for objects with significant detections of $M_{g}$ ) vs. $M_{d}$, showing that the former usually underestimates the dust-based disk mass. (d) $\dot{M}$ vs. $M_{\star}$, showing a hint of a correlation (Muzerolle et al. 2003; Alcalá et al. 2016) contaminated by large scatter. (e) $r_{\text {out }}$ vs. $M_{\star}$, showing no correlation. (f) $M_{d}$ vs. $M_{\star}$, exhibiting weak correlation (Pascucci et al. 2016) with a lot of scatter.

measured $M_{\text {dust }}$ and $\dot{M}$. Out of these disks, 18 also have significant measurements of the gas mass based on CO line measurements. Two disks-Sz84 and MYLup-fall in the transitional disk category (Alcalá et al. 2016). The parameters of all 26 systems are listed in Table 1 . We adopt $M_{\text {dust }}$ and disk sizes from Ansdell et al. (2016), gas masses $M_{g}$ from Miotello et al. (2016b), and $\dot{M}$ and stellar parameters from Alcalá et al. (2014, 2016).

For simplicity, in this study we associate the outer disk radius $r_{\text {out }}$ with the semimajor axis obtained in Ansdell et al. (2016) by simple Gaussian fitting of the resolved continuum intensity pattern. This alone may introduce a systematic uncertainty in the determination of $r_{\text {out }}$ at the level of tens of percent. An even more serious error may arise from the possible difference between the radii of the gas and dust disks, evidence for which has been found in a number of systems (Andrews et al. 2012; Cleeves et al. 2016; Walsh et al. 2016). We discuss the impact of the $r_{\text {out }}$ uncertainty on our results in Section 4.1.

We show some characteristics of our systems in Figure 1. We display with different symbols objects that have characteristic accretion time $M_{d} / \dot{M}$ shorter (open) or longer (filled) than $10 \mathrm{Myr}$ (we use this convention in other figures as well). Even though in this work we prefer to be unbiased by the ages of individual systems, the Lupus objects are unlikely to be older than $10 \mathrm{Myr}$ (Alcalá et al. 2014). In the framework of the viscous evolutionary picture the existence of objects with $M_{d} / \dot{M}>10$ Myr would imply the breakdown of the assumption of the disk spreading in a self-similar fashion. This suggests that such objects (seven in our sample) should be treated with caution. We also single out one system, 2MASS $\mathrm{J} 16081497-3857145$, which is close to the brown dwarf regime and is different from the rest of the sample (shown as a red filled hexagon).

The top panel shows that, unlike Manara et al. (2016), we do not observe a significant correlation between $M_{d}$ and $\dot{M}$ (see Table 2 for the statistical parameters of correlations shown in our plots: Pearson correlation coefficient $\rho$, Spearman's rank correlation coefficient $r_{s}$, and $p$-value-probability of the null hypothesis that the two variables have zero correlation). This is most likely explained by the modest size of our sample 


\begin{tabular}{|c|c|c|c|c|c|c|c|c|}
\hline Name & $L_{\star}\left(L_{\odot}\right)$ & $R_{\star}\left(R_{\odot}\right)$ & $M_{\star}\left(M_{\odot}\right)$ & $\lg \left[\dot{M} /\left(M_{\odot} \mathrm{yr}^{-1}\right)\right]$ & SMA $(\operatorname{arcsec})$ & $M_{\text {dust }}\left(M_{\oplus}\right)$ & $M_{g}\left[\min \left(M_{g}\right), \max \left(M_{g}\right)\right]\left(M_{J}\right)$ & $d(\mathrm{pc})$ \\
\hline Sz65 & $0.8318 \pm 0.3623$ & $1.84 \pm 0.40$ & $0.76 \pm 0.18$ & $-9.61 \pm 0.42$ & $0.171 \pm 0.002$ & $15.1559 \pm 0.0752$ & $0.64[0.2,1.5]$ & 150 \\
\hline Sz68 & $5.1286 \pm 2.1919$ & $3.14 \pm 0.67$ & $2.13 \pm 0.33$ & $-8.42 \pm 0.41$ & $0.159 \pm 0.002$ & $35.3387 \pm 0.1081$ & $0.68[0.2,1.5]$ & 150 \\
\hline Sz69 & $0.0880 \pm 0.0410$ & $0.97 \pm 0.22$ & $0.19 \pm 0.03$ & $-9.48 \pm 0.41$ & $0.092 \pm 0.012$ & $3.9858 \pm 0.0658$ & $0.034[0.018,0.07]$ & 150 \\
\hline Sz71 & $0.3090 \pm 0.1420$ & $1.43 \pm 0.33$ & $0.42 \pm 0.11$ & $-9.06 \pm 0.42$ & $0.558 \pm 0.003$ & $39.0213 \pm 0.1481$ & $0.096[0.07,0.3]$ & 150 \\
\hline Sz72 & $0.2520 \pm 0.1160$ & $1.29 \pm 0.30$ & $0.38 \pm 0.09$ & $-8.66 \pm 0.42$ & $0.094 \pm 0.012$ & $3.3137 \pm 0.0658$ & $\ldots$ & 150 \\
\hline Sz73 & $0.4190 \pm 0.1930$ & $1.35 \pm 0.31$ & $0.82 \pm 0.16$ & $-8.18 \pm 0.41$ & $0.245 \pm 0.01$ & $7.1514 \pm 0.1293$ & & 150 \\
\hline Sz83 & $1.3130 \pm 0.6050$ & $2.39 \pm 0.55$ & $0.75 \pm 0.19$ & $-7.19 \pm 0.42$ & $0.379 \pm 0.001$ & $100.3265 \pm 0.1692$ & $1.5[0.48,4.0]$ & 150 \\
\hline Sz84 (td) & $0.1220 \pm 0.0560$ & $1.21 \pm 0.28$ & $0.18 \pm 0.03$ & $-9.27 \pm 0.41$ & $0.392 \pm 0.006$ & $7.6708 \pm 0.094$ & $0.11[0.06,0.22]$ & 150 \\
\hline Sz88A & $0.4880 \pm 0.2250$ & $1.61 \pm 0.37$ & $0.57 \pm 0.15$ & $-8.14 \pm 0.42$ & $0.102 \pm 0.02$ & $3.7351 \pm 0.1253$ & & 200 \\
\hline Sz90 & $0.6607 \pm 0.2845$ & $1.64 \pm 0.36$ & $0.79 \pm 0.17$ & $-8.68 \pm 0.41$ & $0.143 \pm 0.011$ & $9.1205 \pm 0.1922$ & $0.056[0.035,0.1]$ & 200 \\
\hline Sz98 & $2.5119 \pm 1.0755$ & $3.20 \pm 0.69$ & $0.74 \pm 0.20$ & $-7.26 \pm 0.42$ & $0.974 \pm 0.006$ & $99.1394 \pm 0.5933$ & $0.066[0.04,0.1]$ & 200 \\
\hline Sz103 & $0.1880 \pm 0.0870$ & $1.41 \pm 0.30$ & $0.25 \pm 0.03$ & $-9.04 \pm 0.41$ & $0.122 \pm 0.012$ & $4.8214 \pm 0.117$ & & 200 \\
\hline Sz108B & $0.1514 \pm 0.0813$ & $1.33 \pm 0.36$ & $0.19 \pm 0.03$ & $-9.45 \pm 0.41$ & $0.236 \pm 0.005$ & $11.1845 \pm 0.1421$ & $0.65[0.2,1.5]$ & 200 \\
\hline Sz110 & $0.2760 \pm 0.1270$ & $1.61 \pm 0.37$ & $0.26 \pm 0.03$ & $-8.60 \pm 0.41$ & $0.153 \pm 0.009$ & $6.4341 \pm 0.1212$ & $\cdots$ & 200 \\
\hline Sz113 & $0.0640 \pm 0.0300$ & $0.83 \pm 0.19$ & $0.19 \pm 0.03$ & $-8.85 \pm 0.41$ & $0.118 \pm 0.007$ & $9.3378 \pm 0.1128$ & $\cdots$ & 200 \\
\hline Sz114 & $0.3120 \pm 0.1440$ & $1.82 \pm 0.42$ & $0.23 \pm 0.03$ & $-8.99 \pm 0.41$ & $0.342 \pm 0.002$ & $40.28 \pm 0.1713$ & $0.096[0.065,0.28]$ & 200 \\
\hline Sz129 & $0.3715 \pm 0.1600$ & $1.23 \pm 0.27$ & $0.80 \pm 0.16$ & $-8.41 \pm 0.41$ & $0.458 \pm 0.002$ & $42.5653 \pm 0.1222$ & $0.046[0.03,0.09]$ & 150 \\
\hline Sz130 & $0.1600 \pm 0.0740$ & $1.03 \pm 0.24$ & $0.37 \pm 0.09$ & $-9.15 \pm 0.42$ & $0.246 \pm 0.028$ & $1.4547 \pm 0.0823$ & $0.036[0.011,0.05]$ & 150 \\
\hline Sz131 & $0.1318 \pm 0.0583$ & $1.04 \pm 0.23$ & $0.30 \pm 0.04$ & $-9.25 \pm 0.41$ & $0.116 \pm 0.018$ & $2.0141 \pm 0.0682$ & & 150 \\
\hline MYLup (td) & $0.7762 \pm 0.3315$ & $1.13 \pm 0.24$ & $1.02 \pm 0.13$ & $-9.65 \pm 0.41$ & $0.593 \pm 0.003$ & $41.5524 \pm 0.1786$ & $0.083[0.05,0.21]$ & 150 \\
\hline SSTc2d J154508.9-341734 & $0.0575 \pm 0.0283$ & $0.85 \pm 0.21$ & $0.14 \pm 0.02$ & $-8.41 \pm 0.41$ & $0.173 \pm 0.005$ & $10.874 \pm 0.1175$ & $0.77[0.25,2.0]$ & 150 \\
\hline SSTc2d J160002.4-422216 & $0.1479 \pm 0.0666$ & $1.20 \pm 0.27$ & $0.24 \pm 0.04$ & $-9.69 \pm 0.41$ & $0.749 \pm 0.004$ & $28.1662 \pm 0.1481$ & $0.14[0.11,0.7]$ & 150 \\
\hline 2MASS J16085324-3914401 & $0.3020 \pm 0.1477$ & $1.57 \pm 0.38$ & $0.32 \pm 0.04$ & $-9.80 \pm 0.41$ & $0.08 \pm 0.009$ & $8.1763 \pm 0.117$ & $0.034[0.02,0.07]$ & 200 \\
\hline SSTc2d J161029.6-392215 & $0.1585 \pm 0.0698$ & $1.29 \pm 0.29$ & $0.22 \pm 0.03$ & $-9.82 \pm 0.41$ & $0.238 \pm 0.021$ & $2.9831 \pm 0.1462$ & $0.16[0.1,0.56]$ & 200 \\
\hline SSTc2d J161243.8-381503 & $0.6166 \pm 0.2691$ & $1.91 \pm 0.42$ & $0.47 \pm 0.11$ & $-8.78 \pm 0.42$ & $0.162 \pm 0.008$ & $12.4838 \pm 0.2047$ & $\cdots$ & 200 \\
\hline 2MASS J16081497-3857145 & $0.0087 \pm 0.0047$ & $0.33 \pm 0.09$ & $0.10 \pm 0.02$ & $-10.27 \pm 0.42$ & $0.114 \pm 0.019$ & $3.4761 \pm 0.1253$ & $0.022[0.01,0.045]$ & 200 \\
\hline
\end{tabular}

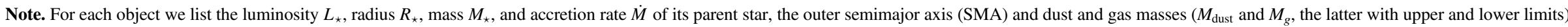

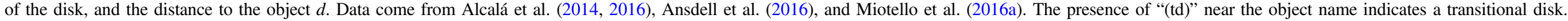


Table 2

Statistical Characteristics of the Data

\begin{tabular}{|c|c|c|c|c|}
\hline Variables & Figures & $\rho$ & $r_{s}$ & $p$-value \\
\hline$\dot{M}-M_{d}$ & 1(a) & 0.446 & 0.3 & 0.137 \\
\hline$r_{\mathrm{out}}-M_{d}$ & 1(b) & 0.7 & 0.631 & $5.5 \times 10^{-4}$ \\
\hline$M_{\mathrm{g}}-M_{d}$ & $1(\mathrm{c})$ & 0.375 & 0.34 & 0.166 \\
\hline$\dot{M}-M_{\star}$ & $1(\mathrm{~d})$ & 0.496 & 0.444 & 0.023 \\
\hline$r_{\text {out }}-M_{\star}$ & $1(\mathrm{e})$ & 0.198 & 0.195 & 0.339 \\
\hline$M_{d}-M_{\star}$ & $1(\mathrm{f})$ & 0.476 & 0.407 & 0.039 \\
\hline$\alpha-M_{d}$ & 3(a) & 0.004 & -0.093 & 0.653 \\
\hline$\alpha-r_{\text {out }}$ & $3(b)$ & -0.01 & -0.045 & 0.828 \\
\hline$\alpha-M_{d} r_{\text {out }}^{-2}$ & $3(\mathrm{c})$ & 0.02 & 0.054 & 0.792 \\
\hline$\alpha-\left(M_{\star} r_{\text {out }}\right)^{1 / 2} M_{d}^{-1}$ & $3(d)$ & 0.18 & 0.209 & 0.306 \\
\hline$\alpha-M_{d}$ & 4(a) & 0.036 & -0.084 & 0.684 \\
\hline$\alpha-M_{d}$ & 4(b) & 0.028 & -0.082 & 0.689 \\
\hline$\alpha-\dot{M}$ & 5 & 0.877 & 0.868 & $9.4 \times 10^{-9}$ \\
\hline$\alpha-\dot{M}$ & $6(a)$ & 0.866 & 0.854 & $2.8 \times 10^{-8}$ \\
\hline$\alpha-\dot{M}$ & $6(\mathrm{~b})$ & 0.868 & 0.858 & $2.1 \times 10^{-8}$ \\
\hline$\alpha-\dot{M}$ & 7 & 0.808 & 0.743 & $4.1 \times 10^{-4}$ \\
\hline$\nu-\dot{M}$ & $8(a)$ & 0.841 & 0.767 & $4.9 \times 10^{-6}$ \\
\hline$\nu-M_{d}$ & $8(b)$ & 0.304 & 0.119 & 0.564 \\
\hline$\nu-r_{\text {out }}$ & $8(c)$ & 0.517 & 0.421 & 0.032 \\
\hline$\alpha-M_{\star}$ & 9 (a) & $0.439(0.354)$ & $0.414(0.341)$ & $0.035(0.095)$ \\
\hline$\alpha-L_{\star}$ & 9 (b) & $0.448(0.326)$ & $0.373(0.295)$ & $0.061(0.153)$ \\
\hline$\alpha-R_{\star}$ & $9(\mathrm{c})$ & $0.45(0.312)$ & $0.335(0.252)$ & $0.094(0.223)$ \\
\hline$\dot{M}-T_{\text {vir }}$ & 10 & 0.177 & 0.235 & 0.247 \\
\hline$\alpha-F_{\mathrm{acc}}$ & 11 & 0.79 & 0.768 & $4.6 \times 10^{-6}$ \\
\hline$M_{d} / \dot{M}-\dot{M}$ & 12(a) & -0.753 & -0.806 & $6.9 \times 10^{-7}$ \\
\hline$M_{d} / \dot{M}-M_{d}$ & $12(b)$ & 0.254 & 0.268 & 0.186 \\
\hline
\end{tabular}

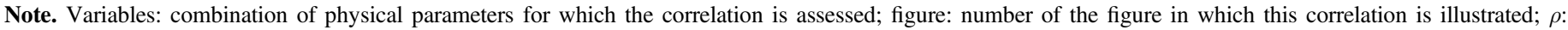

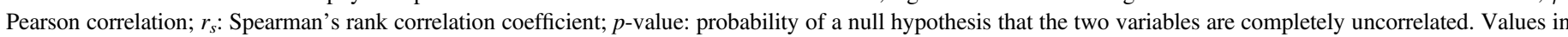
parentheses correspond to the sample with the near-brown-dwarf object 2MASS J16081497-3857145 excluded.

compared to that of Manara et al. (2016) and the significant scatter around the $\dot{M}-M_{\text {dust }}$ correlation found in that work.

On the other hand, we do find an appreciable correlation between the disk size and mass, as Figure 1(b) demonstratesmore extended disks typically have larger dust masses. The best-fit bisector regression (Isobe et al. 1990) describing this correlation is $\lg r_{\text {out }}=(3.05 \pm 0.16)+(0.6 \pm 0.06) \lg M_{d}$ (with $r_{\text {out }}$ and $M_{d}$ measured in au and $M_{\odot}$ ), but there is significant scatter around it. This relation may seem to suggest that the values of the disk surface density at the outer edge $\Sigma\left(r_{\text {out }}\right) \propto M_{d} / r_{\text {out }}^{2}$ should be roughly the same. This might raise a worry that $r_{\text {out }}$, interpreted by Ansdell et al. (2016) as the outer extent of the disk, in fact corresponds to the detection limit of ALMA. However, Figure 3(c) shows that this is not the case, and that $M_{d} / r_{\text {out }}^{2}$ spans almost two orders of magnitude for our sample, thanks to the large scatter in Figure 1(b).

Figure 1(d) demonstrates a hint of a correlation between $\dot{M}$ and stellar mass $M_{\star}$. Such correlation has been known to exist for quite some time (Muzerolle et al. 2003; Alcalá et al. 2016), although in our case it is difficult to see because of large scatter and the small size of our sample. For the same reason we see only a weak correlation between the disk mass $M_{d}$ and $M_{\star}$ in Figure 1(f), which is also expected based on previous studies (Pascucci et al. 2016). We find no correlation between the disk size and $M_{\star}$; see Figure 1(e).

\section{Results}

In Figure 2 we show the histograms for the values of $\alpha$ computed through Equation (4) for different thermodynamic

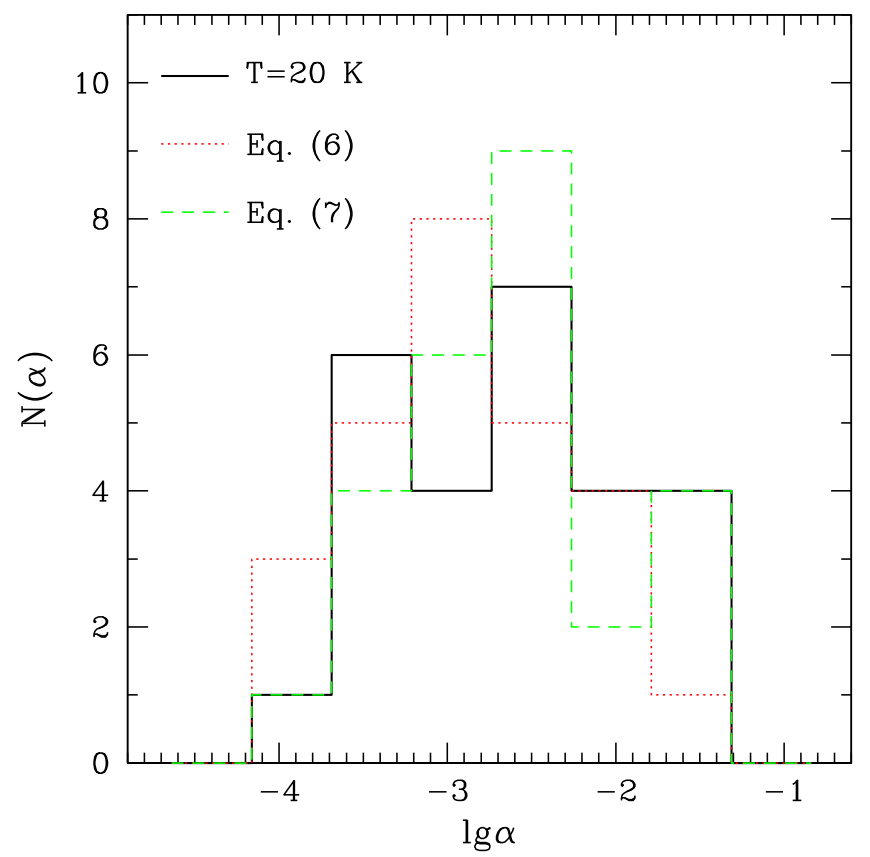

Figure 2. Distribution of the inferred values of $\alpha$, computed via Equation (4). Solid black, dotted red, and dashed green histograms correspond to $T\left(r_{\text {out }}\right)$ prescriptions given by Equations (5)-(7), correspondingly. One can see a large spread in the values of $\alpha$, covering more than two orders of magnitude.

assumptions, as shown in the panel. One can see that different methods of calculating the outer disk temperature do not result in large differences in the values of $\alpha$. Regardless of our 


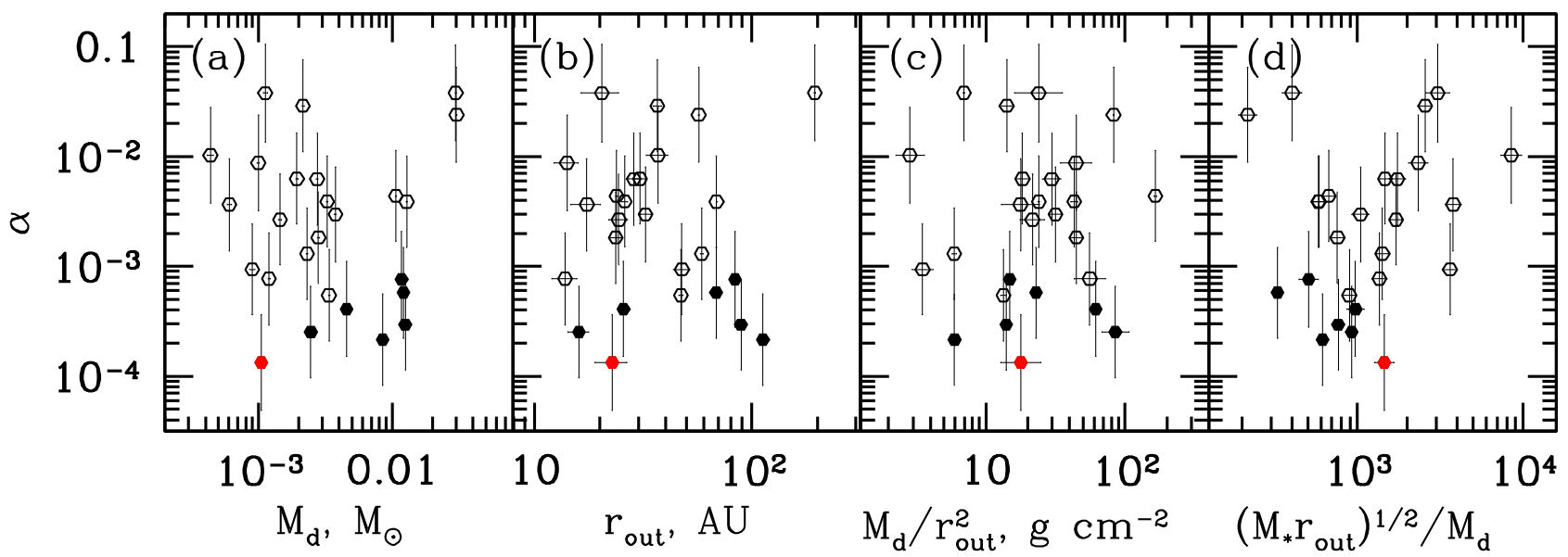

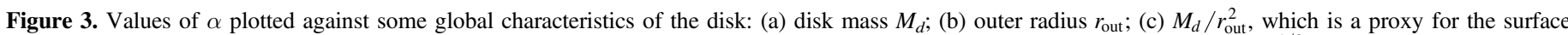

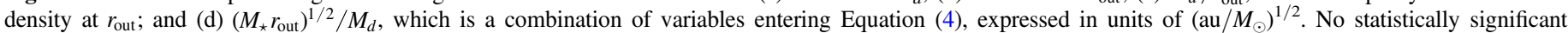
correlations between $\alpha$ and these global variables are found (see Table 2 for quantitative metrics).

assumptions, the distribution of $\alpha$ does not seem to show complicated substructure, roughly consistent with being either peaked (for $T\left(r_{\text {out }}\right)$ given by Equations (6) and (7)) or approximately uniform (for $T\left(r_{\text {out }}\right)=20 \mathrm{~K}$ ).

The most important feature of these distributions is their broad range. Irrespective of the $T\left(r_{\text {out }}\right)$ prescription, we find that in our sample of 26 disks the values of $\alpha$ span more than two orders of magnitude-from $10^{-4}$ to 0.04 . This spread is hardly compatible with the simple idea of a single angular momentum transport mechanism setting the value of $\alpha$, as one would then expect a narrowly peaked distribution of $\alpha$ values. Nor could it be several physical mechanisms operating in different systems (e.g., different instabilities driving the transport), as then one would expect to see more substructure in the distribution of $\alpha$.

As we show in Section 4.1, this spread is unlikely to be forced by the intrinsic scatter or observational errors in our sample. Some of this dispersion can be explained by the fact that all objects with low inferred $\alpha<6 \times 10^{-4}$ have accretion times $M_{d} / \dot{M}>10 \mathrm{Myr}$ (e.g., see Figure 3). As mentioned in Section 2.1 , this likely implies that these systems do not evolve in a self-similar fashion, which makes their $\alpha$ determined via Equation (4) suspect. This, however, cannot explain the twoorders-of-magnitude spread in $\alpha$ for the rest of our objects. Thus, we are left to hypothesize that there must be some other reasons for this behavior of $\alpha$ in different systems, and we try to identify them next.

\subsection{Dependence of $\alpha$ on Global Disk Properties}

What we are calculating via Equation (4), given the observables, is the value of $\alpha$ at $r_{\text {out }}$, which determines the global evolution of the disk. For this reason it is natural to seek possible connection of $\alpha$ with the global variables characterizing the disk on scales $\sim r_{\text {out }}$.

In Figure 3 we plot $\alpha$ computed for $T\left(r_{\text {out }}\right)=20 \mathrm{~K}$ versus the disk mass $M_{d}$; its radial extent $r_{\text {out }} ; M_{d} / r_{\text {out }}^{2}$, which characterizes the surface density at $r_{\text {out }}$; and $\left(M_{\star} r_{\text {out }}\right)^{1 / 2} M_{d}^{-1}$, which appears in Equation (4) together with $\dot{M}$. The errors on $\alpha$ were calculated quadratically from the uncertainties of the observables, as follows from Equation (4). It is clear that these plots do not reveal significant correlations of $\alpha$ with these global variables. This is also confirmed by the quantitative metrics of the possible relations between the pairs of variables shown in Table 2.

One may wonder whether this lack of correlation with the global disk parameters is forced by our simple assumption about the thermal state of the disk, represented by Equation (5). To address this issue, in Figure 4 we show the analog of Figure 3(a), i.e., $\alpha$ versus $M_{d}$, but calculated for $T\left(r_{\text {out }}\right)$ given by Equations (6) and (7). One can see that, again, no correlation is present in the data, implying that this result is robust with regard to our assumptions about the disk temperature structure. To summarize, we do not find any clear dependence of $\alpha$ on the most obvious global characteristics of the disk.

\subsection{Dependence of $\alpha$ on $\dot{M}$}

Effective viscosity computed via Equation (4) depends not only on the global disk characteristics but also on the central mass accretion rate $\dot{M}$. In Figure 5 we plot the effective viscosity $\alpha$ for $T\left(r_{\text {out }}\right)=20 \mathrm{~K}$ versus $\dot{M}$. This figure clearly reveals a strong correlation (Pearson coefficient $\rho(\alpha, \dot{M})=0.877)$ between $\alpha$ and $\dot{M}$. Simple linear bisector regression (Isobe et al. 1990) results in a best-fit line

$$
\lg \alpha=(5.98 \pm 0.91)+(0.97 \pm 0.1) \lg \dot{M}
$$

(with $\dot{M}$ measured in $M_{\odot} \mathrm{yr}^{-1}$ ), which is consistent with a linear dependence. This relation links the broad distribution of $\alpha$ seen in Figure 2 with the spread of $\dot{M}$ in our sample.

The $\alpha-\dot{M}$ correlation is robust with respect to our assumptions about $T\left(r_{\text {out }}\right)$, as further demonstrated in Figure 6. There we again observe that $\dot{M}$ and the effective viscosity parameter computed using Equations (6) and (7) are strongly correlated, despite the different assumptions about disk thermodynamics.

Correlation persists even if we use the CO-based gas masses $M_{g}$, available for 18 objects in our sample, instead of the dustbased masses $M_{d}$ when computing $\alpha$. This is illustrated in Figure 7. The spread in the values of $\alpha$ measured this way is considerably larger than in Figures 5-6, and the best-fit line is significantly offset from relation (8), illustrating the problem 


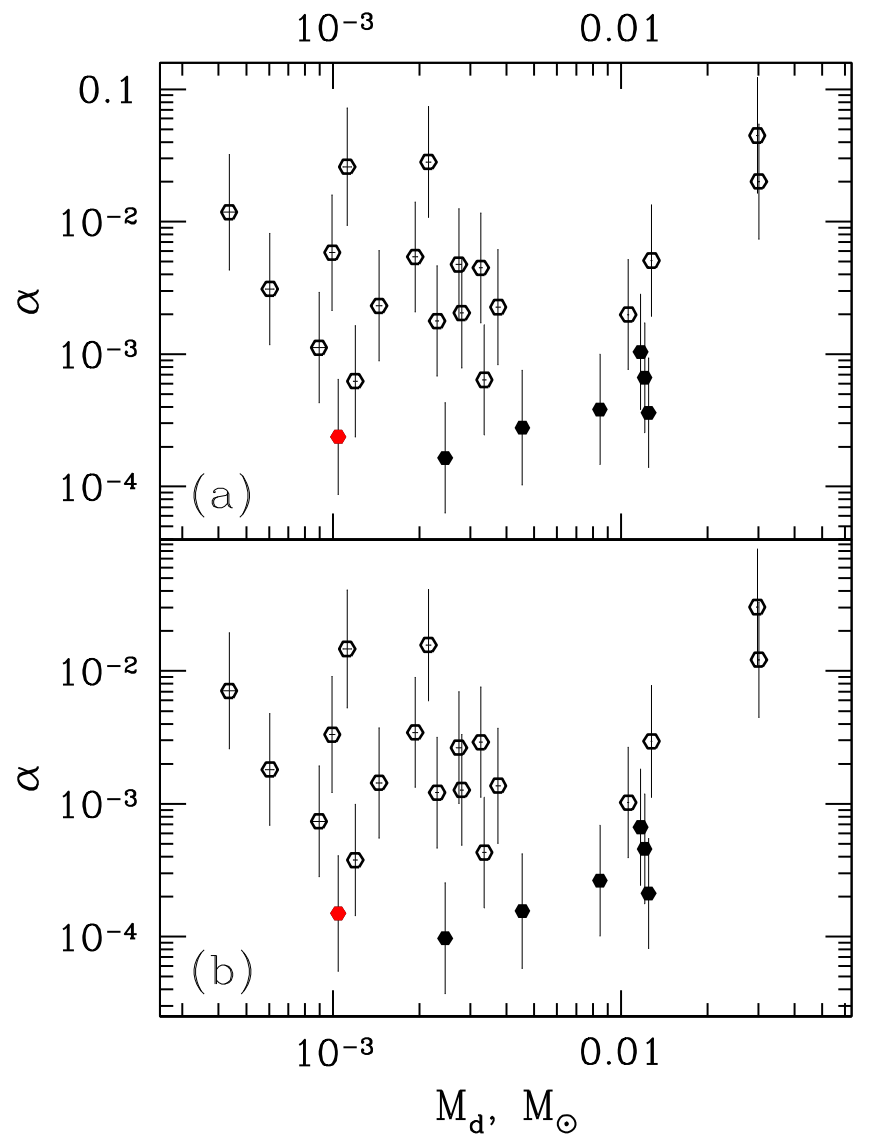

Figure 4. Values of $\alpha$ computed assuming $T$ ( $r_{\text {out }}$ ) given by (a) Equation (6) and (b) Equation (7), plotted as a function of the disk mass $M_{d}$. No correlations emerge here, in agreement with Figure 3(a).

with the CO-based disk masses (Ansdell et al. 2016; Miotello et al. 2016a).

If we were to take the CO-based masses at face value, we would conclude from Figure 7 that, in the framework of the viscous model for the disk evolution based on Equations (1)(3), some systems require $\alpha \gtrsim 1$. As such values of $\alpha$ are unlikely, this could, again, demonstrate the problem with the disk mass determinations based on the $\mathrm{CO}$ line emission.

The existence of a tight correlation between $\alpha$ and $\dot{M}$ is a nontrivial and rather unexpected result. Indeed, if the angular momentum transport in the disk were effected by a mechanism characterized by a unique value of $\alpha$, then Figure 5 would look very differently, with $\alpha$ clustering around a well-defined value regardless of $\dot{M}$, and the slope of the $\alpha(\dot{M})$ relation being close to zero, as illustrated by the cyan line in this figure. The $\alpha=10^{-2}$ corresponding to this line is for illustrative purposes, although this value has been suggested by previous studies (Hartmann et al. 1998). In that case the variation of $\dot{M}$ would have been exactly compensated by the variation of $\Omega r_{\text {out }}^{2} /\left(M_{d} T\right)$. Figure 3(d) shows that the latter variable (proportional to $\left.\left(M_{\star} r_{\text {out }}\right)^{1 / 2} M_{d}^{-1}\right)$ exhibits essentially no correlation with $\alpha$, unlike $\dot{M}$ entering expression (4) for $\alpha$ in an identical fashion. Thus, the very fact that a strong $\alpha-\dot{M}$ correlation exists tells us something interesting.

We also explored the behavior of the dimensional kinematic viscosity $\nu \approx r^{2} \dot{M} / M_{d}$, which plays the role of a diffusion coefficient for viscous spreading of the disk; see Figure 8. One possible advantage of using $\nu$ instead of $\alpha$ is that its determination does not involve assumptions about the

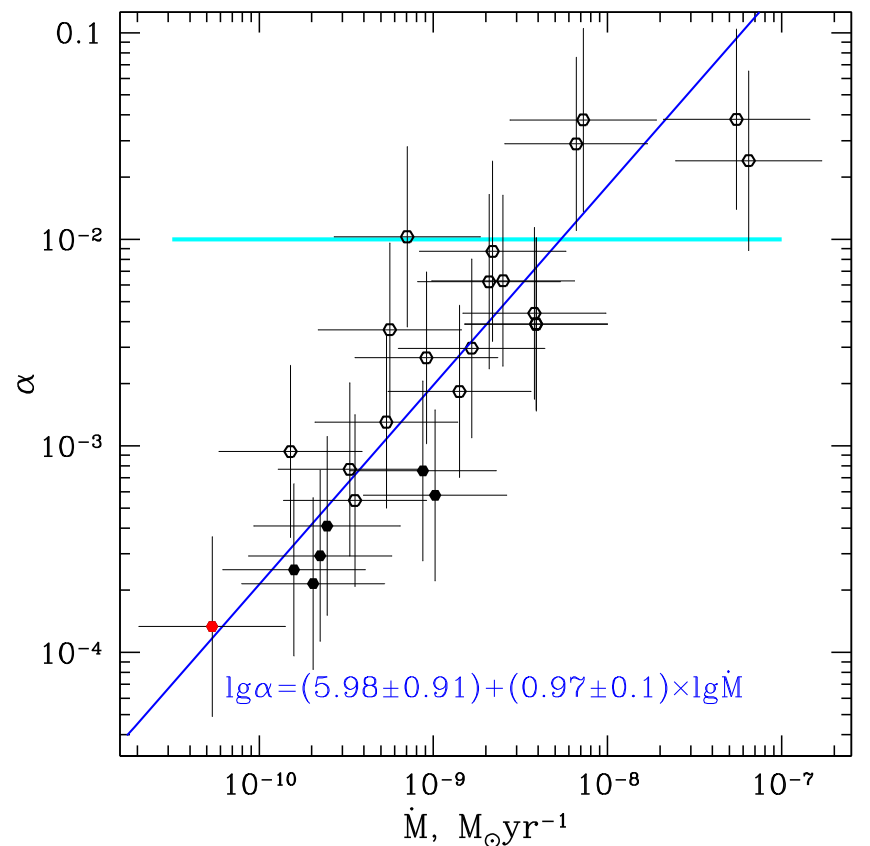

Figure 5. Effective viscosity $\alpha$, computed for $T=20 \mathrm{~K}$ (Equation (5)), as a function of the central mass accretion rate $\dot{M}$. One can clearly see a strong correlation between the two variables. The blue line is the best fit to the data given by Equation (8). The horizontal cyan line is the dependence, around which the data points would be expected to cluster if the angular momentum transport were characterized by a single value of $\alpha$ (taken to be equal to $10^{-2}$ for illustrative purposes). Such clustering is clearly not exhibited by our sample, necessitating modifications to the simple picture of the viscous evolution of the protoplanetary disks.

thermodynamic properties of the disk. One can see that $\nu$ is also strongly correlated with $\dot{M}$ (although the spread around the best-fit line is larger than in Figures 5-6), while at the same time being independent of either $M_{d}$ or $r_{\text {out }}$. This again suggests that there is a certain causal relation between $\dot{M}$ and the inferred disk viscosity.

\subsection{Dependence of $\alpha$ on Stellar Properties}

Having found correlation of $\alpha$ with $\dot{M}$, which is a local characteristic measured at the star, we also checked whether $\alpha$ could have some relation to other stellar parameters.

In Figure 9 we examine this possibility, finding no significant correlations between $\alpha$ and the stellar mass, luminosity, or radius. Weak correlations that may be present in the full data set vanish when we remove the brown-dwarflike object 2MASS J16081497-3857145 (which has very distinct properties and strongly affects covariances between the variables) from the sample.

This lack of correlation is not surprising from the physical point of view, as one may expect only a relatively weak effect of $M_{\star}$ (e.g., through local shear, proportional to $M_{\star}^{1 / 2}$ ) or $L_{\star}$ (on which the disk temperature might depend) on the global disk properties.

\section{Discussion}

Having established a close relation between the mass accretion rate onto the central star $\dot{M}$ and the inferred value of $\alpha$ on the global scale of the disk, we now seek to understand the implications of this finding. When doing this, it is also important to keep in mind the lack of any significant 


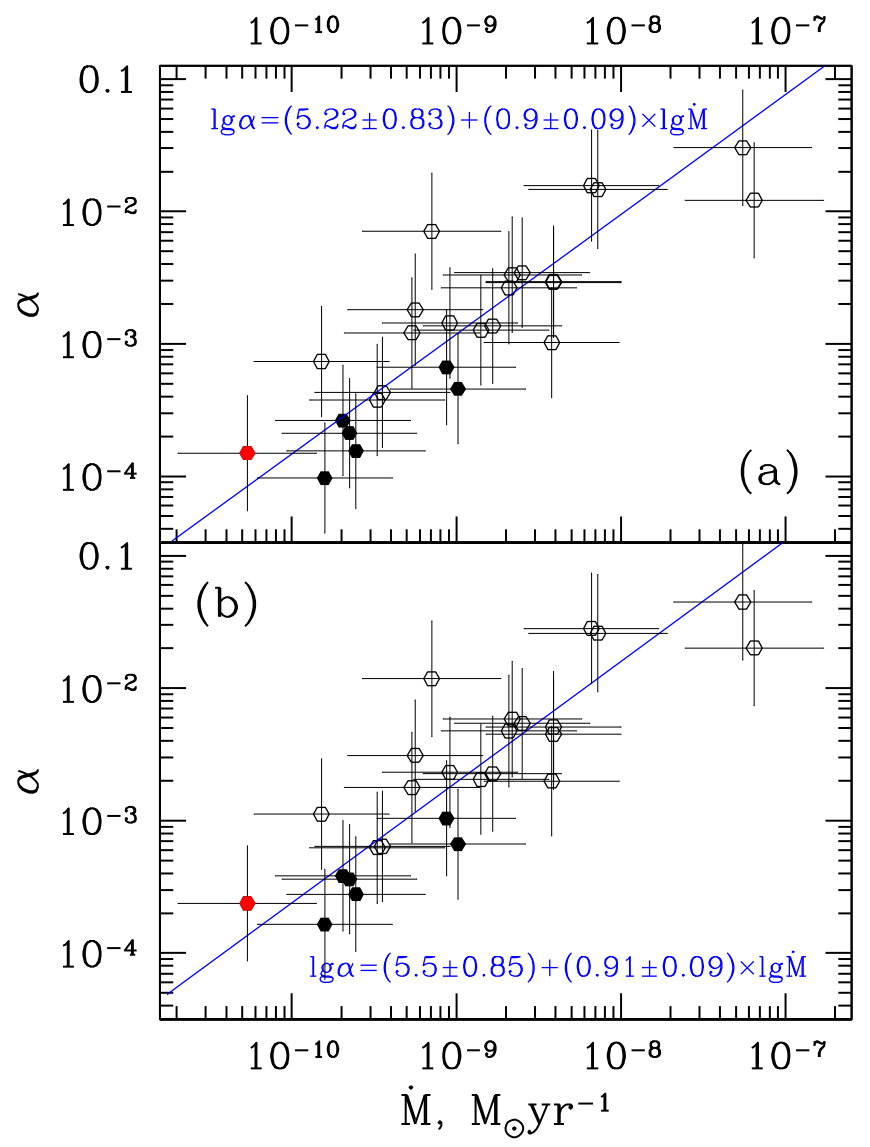

Figure 6. Correlations between the central mass accretion rate $\dot{M}$ and $\alpha$, computed using different assumptions about disk temperature: (a) $T(r)$ given by Equation (6), and (b) $T(r)$ given by Equation (7). Correlation between $\dot{M}$ and $\alpha$ clearly persists in both cases.

correlations of $\alpha$ with other obvious characteristics of the system, be it global (like $M_{d}$ or $r_{\text {out }}$ ) or local, stellar (e.g., $M_{\star}$, or $L_{\star}$ ).

There are different ways in which such a correlation could emerge. First, it may result from various systematic effects related to the measurement of the observables (Section 4.1). Second, there may be a physical reason for the correlation. This would be the case if, for example, some processes related to accretion of gas onto the stellar surface are able to influence the value of $\alpha$ globally, on scales $\sim r_{\text {out }}$ (Section 4.2). Alternatively, the value of the $\alpha$-parameter may depend on some yetunidentified property of the protoplanetary disk, resulting in observed spread, and giving rise to a variation of $\dot{M}$ (Section 4.3). Third, the $\alpha-\dot{M}$ relation (8) may simply reflect the way in which $\dot{M}$ enters the determination of $\alpha$ in Equation (4), with $\dot{M}$ being, in fact, largely unrelated to the global disk characteristics. This would be the case if the central $\dot{M}$ were decoupled from the global accretion rate set by the disk properties, e.g., as a result of some instability operating in the inner disk, or mass accumulation in a dead zone (Section 4.4). Decoupling would also be natural if the angular momentum transport in protoplanetary disks does not have a diffusive character (Section 4.5) and is not characterized by Equations (1)-(3).

We now examine each of these possibilities in detail.

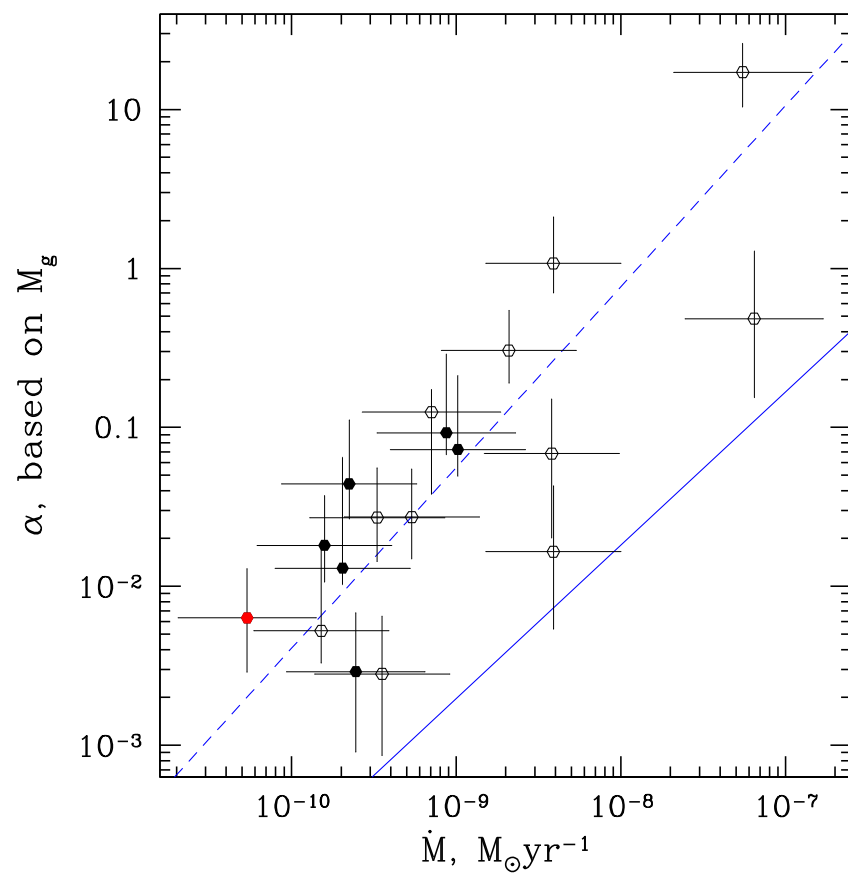

Figure 7. $\alpha$ computed using gas masses $M_{g}$ inferred from the CO line observations, instead of the dust-based masses $M_{d}$ computed using the continuum submillimeter emission (displayed in Figures 5-6), shown as a function of $\dot{M}$. Despite the use of a different tracer of the disk mass, the $\alpha-\dot{M}$ correlation (dashed line) is still present at high significance. The solid line corresponds to correlation (8), which is clearly offset from the best fit for CObased $\alpha$ and $\dot{M}$.

\subsection{Observational Biases}

Our calculation of $\alpha$ involves several observables $-\dot{M}, M_{d}$, $r_{\text {out }}$-and we need to make sure that the origin of the $\alpha-\dot{M}$ correlation is not related to the possible systematic biases in their measurement. We do this next for each of these variables.

\subsubsection{Uncertainty in $\dot{M}$}

Determination of stellar $\dot{M}$ is a challenging task, which was accomplished in Alcalá et al. $(2014,2016)$ by measuring the UV excess above the stellar photospheric emission. A variety of factors, including the differences between the stellar evolution tracks computed by different groups, contribute to the uncertainty in the subsequent derivation of $\dot{M}$, which we conservatively adopted to be about 0.4 dex (Alcalá et al. 2016). However, it is not easy to see how they could enforce a systematic (and not random) correlation as given by Equation (8).

One way to do this might involve the unobserved portion of the accretion luminosity, which could skew the $\dot{M}$ determination in a systematic way. Indeed, it may be the case that in many systems most of the accretional energy $\approx G M_{\star} \dot{M} / R_{\star}$ is re-emitted in the (high-energy) spectral region inaccessible to ground-based instruments. In that case the accretion luminosity measured from the ground would account for only a small fraction of the bolometric accretion flux. If gas accretion is mediated by a stellar magnetosphere, which truncates the disk, then one may expect (Calvet \& Gullbring 1998) the discrepancy in $\dot{M}$ determination to correlate with the virial temperature $T_{\text {vir }}=(\mu / k) G M_{\star} / R_{\star}$ of the gas striking the stellar surface in freefall- the higher $T_{\text {vir }}$ would shift emission from accretion shock to shorter wavelengths and result in a more 


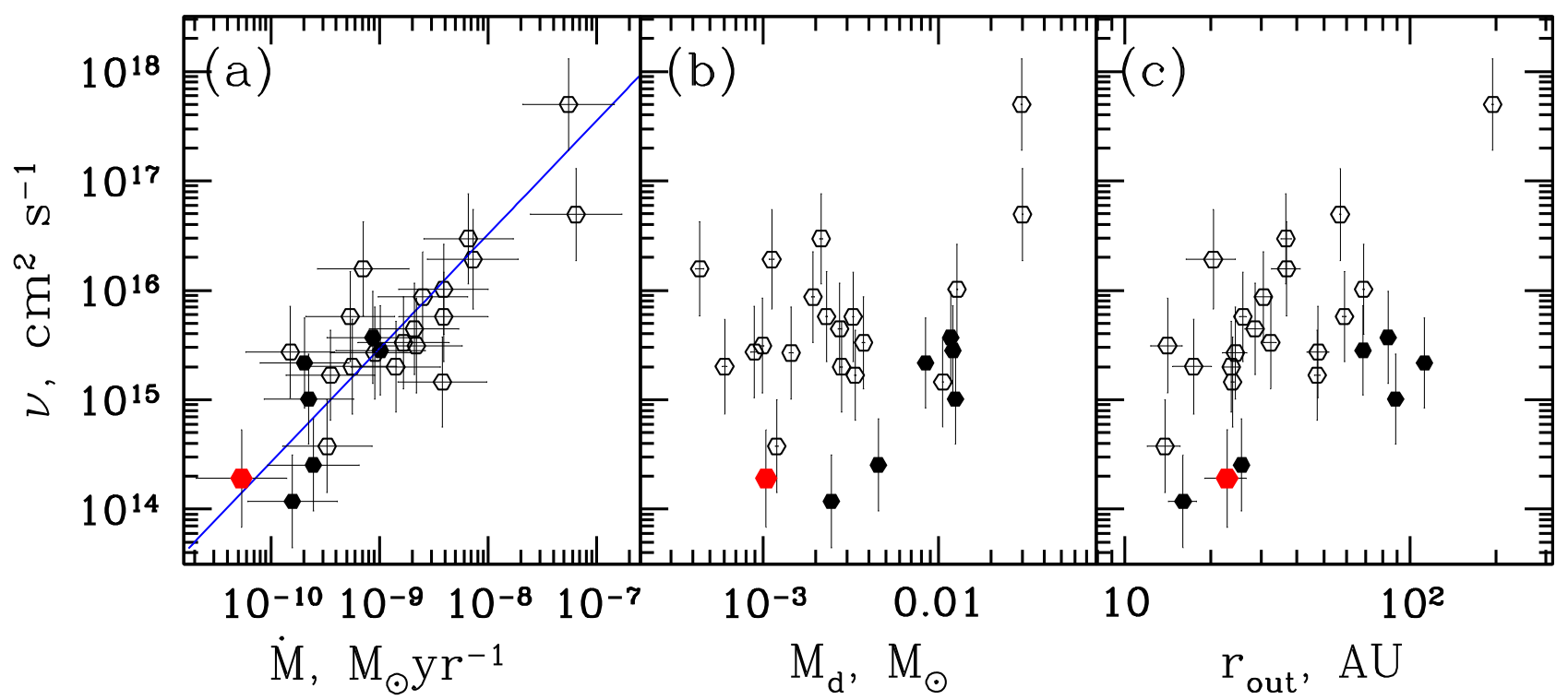

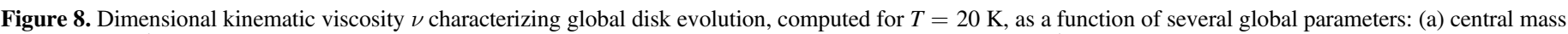

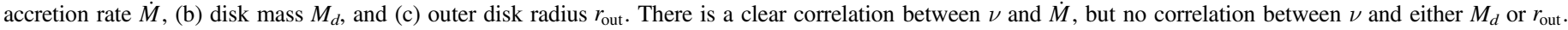

severe underestimate of $\dot{M}$. This deviation of $\dot{M}$ from its true value would then lead to an underestimate of $\alpha$ inferred through Equation (4), perfectly correlated with the biased estimate of $\dot{M}$.

To address this issue, in Figure 10 we plot $\dot{M}$ versus the virial temperature calculated using stellar parameters from Table 1. One can see no correlation of the kind suggested above, with systems having higher $T_{\text {vir }}$ not showing systematically lower values of $\dot{M}$. The two variables appear completely uncorrelated in our sample. This suggests that the determination of $\dot{M}$ does not suffer from the bias related to the unobserved accretion luminosity.

\subsubsection{Uncertainty in $r_{\mathrm{out}}$}

In this work we also implicitly assumed that $r_{\text {out }}$, obtained in Ansdell et al. (2016) by fitting a Gaussian to the observed intensity pattern, is the true outer disk radius, which encloses its full mass. One may worry that, in fact, this radius corresponds to the sensitivity limit of ALMA and in reality the disk extends beyond $r_{\text {out }}$, so that both $r_{\text {out }}$ and $M_{d}$ underestimate their true values. However, Figure 3(c) demonstrates that this is not the case: the values of $M_{d} / r_{\text {out }}^{2}$ proportional to the surface brightness of the outer disk do not cluster around a single value (which could be interpreted as the sensitivity limit of observations), but rather extend over almost two orders of magnitude.

A potentially more serious issue with $r_{\text {out }}$ may arise in systems with different apparent sizes of the gas and dust disks. Evidence for this discrepancy has been found recently in TW Hya (Andrews et al. 2012), IM Lup (Cleeves et al. 2016), and HD 97048 (Walsh et al. 2016), with the dust continuum emission being radially more centrally concentrated by a factor of 2-3 than the gaseous disk emitting in ${ }^{12} \mathrm{CO}$ lines. This has been interpreted as the evidence for the radial inward drift of solids in these disks (Birnstiel \& Andrews 2014), which decouples radial distributions of the gas and dust. If this interpretation is correct, then the dust masses would still properly reflect the full disk mass, but the size of the main mass reservoir (gas disk) would be underestimated by a factor of several. Although this issue should be further explored observationally for our Lupus sample, we believe that it is unlikely to affect our main conclusions for the following reasons.

First, the inferred $\alpha$ depends on $r_{\text {out }}$ rather weakly, e.g., as $\alpha \propto r_{\text {out }}^{1 / 2}$ if $T=20 \mathrm{~K}$; see Equation (4). Thus, a possible underestimate of $r_{\text {out }}$ by a factor of 2-3 would not explain the broad distribution of the inferred values of $\alpha$. Second, it is not clear that the gas disk sizes based on ${ }^{12} \mathrm{CO}$ measurements represent the radii where most of the gas mass is concentrated (which is what the actual $r_{\text {out }}$ should correspond to). Because of the optical thickness of the ${ }^{12} \mathrm{CO}$ lines, it is generally believed that the $\mathrm{CO}$ isotopologues are better tracers of the gas mass distribution than the ${ }^{12} \mathrm{CO}$ molecule. And the sizes of regions emitting in ${ }^{13} \mathrm{CO}$ and $\mathrm{C}^{18} \mathrm{O}$ lines tend to be less discrepant with the dust-continuum-based radii than the ones based on ${ }^{12} \mathrm{CO}$ emission (Cleeves et al. 2016; Schwarz et al. 2016). This statement seems to hold in our sample too (Ansdell et al. 2016), based on the disk images obtained using different tracers.

\subsubsection{Uncertainty in $\mathrm{M}_{\mathrm{d}}$}

Finally, we discus the effect of the uncertainty in the disk mass measurement. Miotello et al. (2016a) derived more accurate dust-continuum-based masses of the disks from the sample of Ansdell et al. (2016) using detailed radiative transfer calculations of the thermal structure of the disk (instead of assuming a single $T=20 \mathrm{~K}$ as in Ansdell et al. 2016). They found that Ansdell et al. (2016) systematically overestimate $M_{d}$ by about a factor of 2 for $M_{d} \lesssim 10^{-2} M_{\odot}$. However, this bias would simply uniformly shift our $\alpha-\dot{M}$ relation, without affecting its scatter or slope. A similar effect would be caused by the possibility of an inward drift of solids (see Section 4.1.2), which tends to decrease the gas-to-dust ratio $\chi$ in the disk region probed by the submillimeter continuum measurements. However, such bias would just shift down (roughly uniformly) the disk mass enclosed within the dust disk radius, without breaking the $\alpha-\dot{M}$ correlation or increasing the spread of $\alpha$.

Moreover, Equation (4) remains valid even if $r_{\text {out }}$ and $M_{d}$ do not characterize the full disk: as long as $M_{d}(<r)$ accounts for 


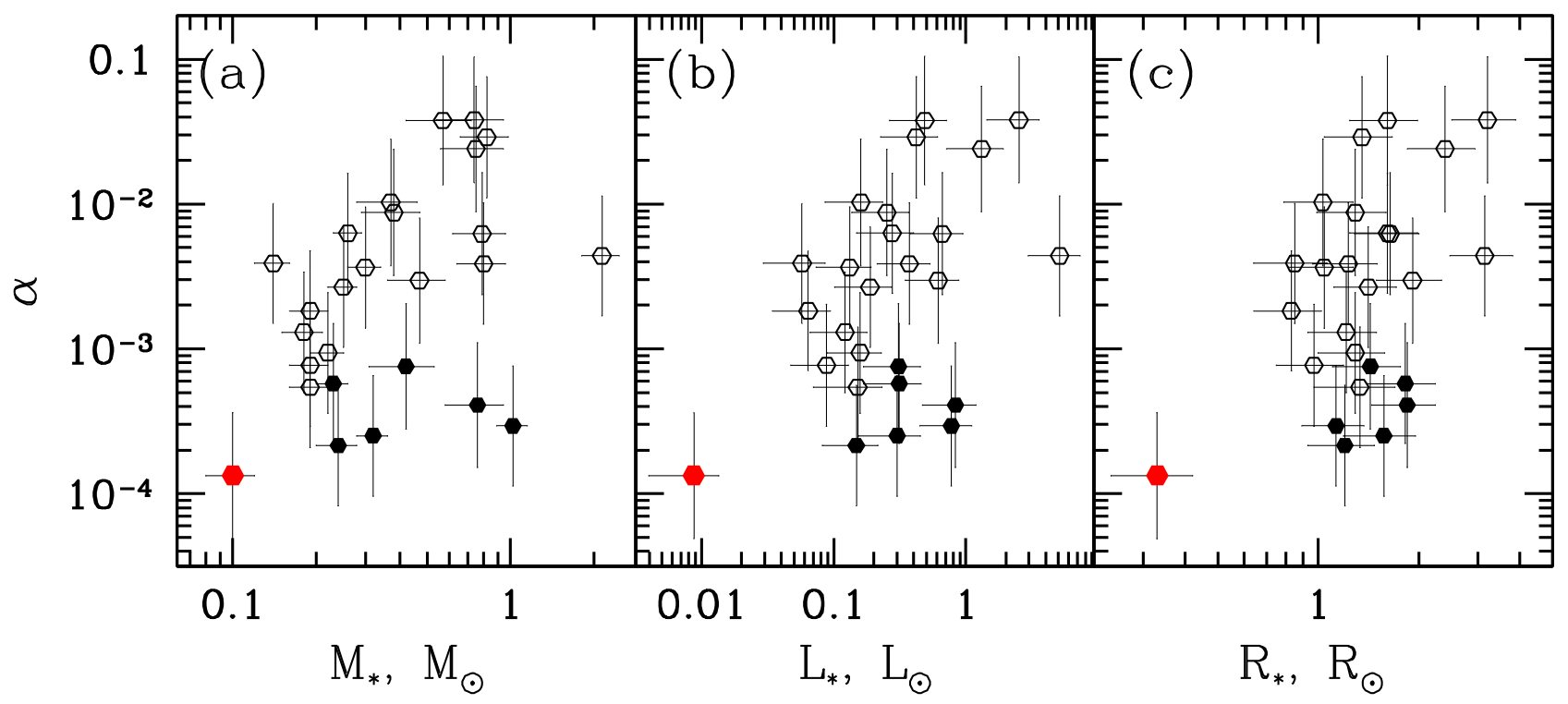

Figure 9. Effective viscosity $\alpha$, computed for $T=20 \mathrm{~K}$, plotted vs. stellar parameters: (a) stellar mass $M_{\star}$, (b) luminosity $L_{\star}$, and (c) radius $R_{\star}$. No clear correlations are present in the data, especially when the near-brown-dwarf object 2MASS J16081497-3857145 (red filled hexagon) is not included in the sample.

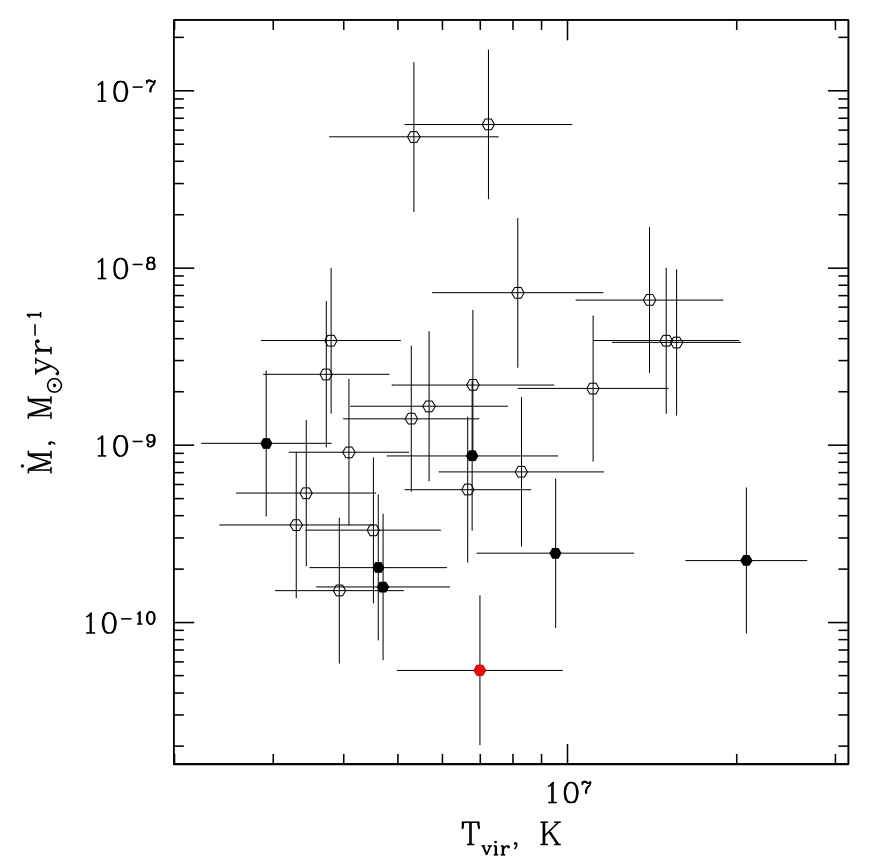

Figure 10. Stellar $\dot{M}$ plotted vs. the virial temperature at the stellar surface $T_{\text {vir }}$. No obvious correlation is seen in the data, demonstrating the lack of biases related to the unobserved fraction of the accretion luminosity.

the full disk mass enclosed within some radius $r$, their values can be used instead of $M_{d}$ and $r_{\text {out }}$ for the determination of $\alpha$ via Equation (4).

Based on this discussion, we conclude that observational uncertainties and biases are unlikely causes of correlation (8) and can hardly account for the full spread in the inferred values of $\alpha$ seen in Figure 2 .

\section{2. $\dot{M}$ Setting $\alpha$}

Another possibility for the origin of the $\alpha-\dot{M}$ correlation is that the central $\dot{M}$ has a direct physical effect on $\alpha$. It is difficult to see how this connection can be realized in practice, since $\alpha$

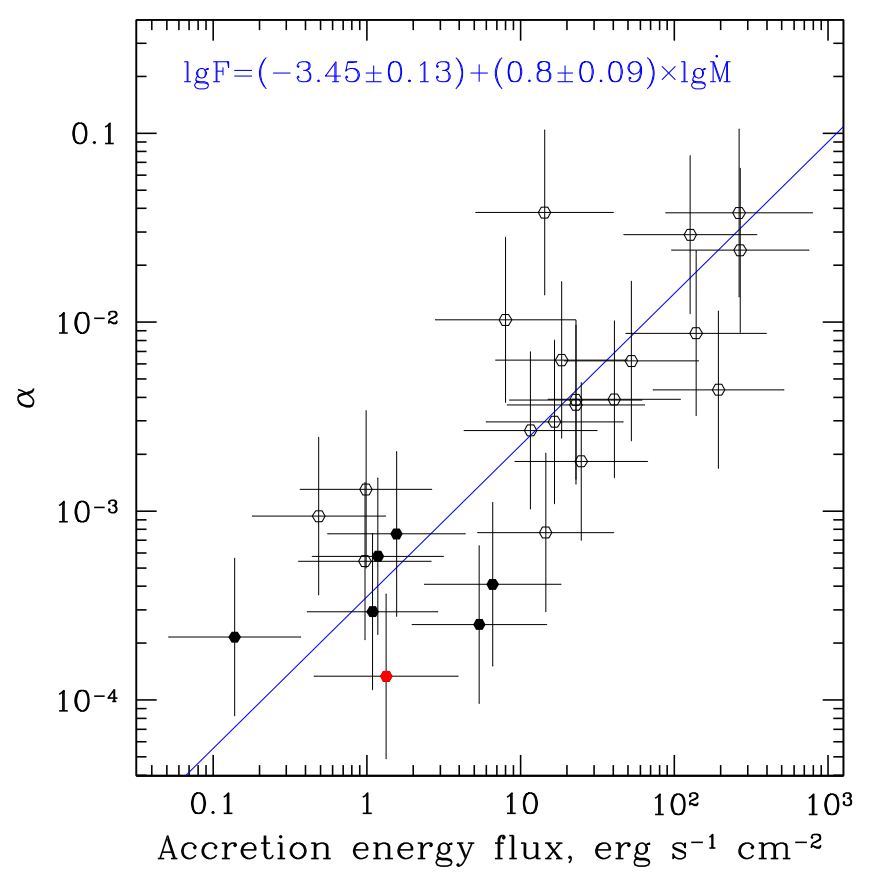

Figure 11. Effective viscosity plotted as a function of the accretion energy flux at the outer radius of the disk, $F=G M_{\star} \dot{M} /\left(4 \pi R_{\star} r_{\text {out }}^{2}\right)$.

is set by the disk physics on global scales, while $\dot{M}$ is a local property, characterizing the innermost region of the disk.

One possibility for establishing this connection is if the accretion energy release at the stellar surface has a direct impact on the value of $\alpha$ on global scales. This may be the case if the value of $\alpha$ depends on the degree of ionization (as may be expected for the nonideal MRI), and the accretional luminosity plays a major role in determining the ionization balance in the outer disk. If that were the case, one would expect to see a correlation between the global $\alpha$ and the accretion energy flux $F=G M_{\star} \dot{M} /\left(4 \pi R_{\star} r_{\text {out }}^{2}\right)$ at $r=r_{\text {out }}$.

Figure 11 demonstrates that such correlation does indeed exist. However, it shows larger scatter around the best-fit line than the correlation in Figure 5. This would not be expected if 
it were $F$ rather than $\dot{M}$ alone being the real culprit behind the $\alpha-\dot{M}$ correlation. Moreover, it is also unlikely that the spectral range used for inferring $\dot{M}$ (longward of $310 \mathrm{~nm}$; Alcalá et al. 2016) dominates the ionization balance of the disk. Nor is it clear that the accretion energy release provides a major contribution to the flux of ionizing photons impinging on the disk (Glassgold et al. 2000). Furthermore, it is not obvious why this physical mechanism should give rise to an $\alpha(F)$ dependence with a slope so close to unity.

One final argument against a physical effect of the central $\dot{M}$ on the global value of $\alpha$ is that the disk with $\alpha(F) \propto F^{0.8} \propto r^{-1.6}$ dependence, suggested by the best fit in Figure 11, should have a rather unusual structure. Indeed, inside $r_{\text {out }}$ the disk should converge to a constant $\dot{M}$ structure, meaning that $\Sigma \propto \dot{M} / \nu \propto r^{0.1} T^{-1}$, where we used Equation (2) for $\nu$ and took $\alpha \propto r^{-1.6}$. Since the disk temperature $T$ does not increase with $r$, this would mean that $\Sigma$ should be an increasing function of $r$. This conclusion is hardly compatible with our understanding of the protoplanetary disk structure.

For all these reasons we do not find the direct physical effect of stellar $\dot{M}$ on $\alpha$ to be a plausible explanation of the $\alpha-\dot{M}$ correlation.

\section{3. $\alpha$ Setting $\dot{M}$}

A physical connection between $\alpha$ and $\dot{M}$ may also emerge in the direction opposite to that considered in Section 4.2, with $\alpha$ directly affecting $\dot{M}$. Such a connection is rather natural in light of Equation (4). However, in the conventional picture $\alpha$ has a unique value, which is incompatible with the distribution shown in Figure 2. Thus, the broad range of $\alpha$ in the observed sample has to be caused by some additional environmental parameter, which controls angular momentum transport and allows $\alpha$ to vary over almost three orders of magnitude. And then the $\alpha-\dot{M}$ correlation would naturally emerge from Equation (4), with the distribution of $\alpha$ directly translating into the broad range of the $\dot{M}$ values.

The hidden parameter controlling $\alpha$ cannot be one of the global disk variables- $M_{d}, r_{\text {out }}$, global surface density-as Figure 3 shows no correlation of $\alpha$ with them. The cooling time of the disk, which directly depends on these global disk characteristics, also cannot be the controlling parameter. This likely excludes the vertical shear instability (Urpin \& Brandenburg 1998), which sensitively depends on the local cooling time (Stoll \& Kley 2014; Lin \& Youdin 2015), from being a candidate for driving the viscous evolution of the protoplanetary disks.

At the same time, there are a variety of possible controlling parameters if transport in the disk on scales $\sim r_{\text {out }}$ is driven by the (nonideal) MRI. They include (but are not limited to) thermal gas pressure (Ross et al. 2016), ionization fraction (Jin 1996; Fleming et al. 2000; Bai \& Stone 2011), strength of the magnetic field in the disk (Bai \& Stone 2011), or its geometry (Simon et al. 2013a, 2013b). Most of these physical characteristics are difficult to determine observationally at the moment.

The idea of global (but variable from object to object) $\alpha$ is likely incompatible with the self-similar viscous evolution of the disk in systems with long $M_{d} / \dot{M} \gtrsim 10 \mathrm{Myr}$. Selfsimilarity implies that accretion time should be equal to the system age, and Lupus objects are not that old (Alcalá et al. 2014). This suggests that the assumption of self-similarity is violated for this subset of our objects. Nevertheless, we believe that the variation of $\alpha$ between different objects controlled by an additional parameter is a more plausible way of producing an $\alpha-\dot{M}$ correlation than the one outlined in Section 4.2.

\subsection{Decoupling of Stellar $\dot{M}$ from the Global Mass Accretion Rate}

A correlation between the inferred $\alpha$ and $\dot{M}$ would also naturally emerge if the $\dot{M}$ measured through stellar accretional luminosity is, in fact, unrelated to the global mass accretion rate $M_{d} / t_{\nu}$.

In the standard viscous disk theory the two should be equal, as demonstrated by Equation (3). However, if stellar $M$ is somehow decoupled from the global accretion rate, then Equation (4) would naturally result in a strong linear correlation between the inferred $\alpha$ (unrelated to the real global $\alpha$ ) and $\dot{M}$. This would be true even if the real $\alpha$ set by the physics of the angular momentum transport on scales $\sim r_{\text {out }}$ takes on a unique value. Errors in measuring $\dot{M}$ could lead to this situation, but they would need to be very dramatic (potentially exceeding two orders of magnitude), which is unlikely, as we showed in Section 4.1.

A decoupling between $\dot{M}$ and $M_{d} / t_{\nu}$ (by more than two orders of magnitude, to explain the range of inferred $\alpha$ ) requires a modification of the simple picture of viscous disk accretion. It can arise, for example, if some instability operates in the inner regions of protoplanetary disks, dramatically modulating local $\dot{M}$ compared to its global value set on scales $\sim r_{\text {out }}$. The characteristic timescale for such variability should be substantial for it to have escaped detection until now. One may suspect FUor and EXor outbursts (Audard et al. 2014) to be the known realizations of such an instability. However, one would then expect the distribution of $\alpha$ to be bimodal, with most disks being in quiescence and having low $\alpha$, and a small population of disks undergoing an outburst and having high inferred $\alpha$ (Audard et al. 2014).

Another way of decoupling stellar $\dot{M}$ from the global accretion rate is if the viscous mass flow toward the star accumulates in a substantial mass reservoir at some intermediate radii, e.g., in a dead zone (Gammie 1996). This reservoir should be able to accumulate large amounts of mass, comparable to the total disk mass at the start of its evolution. This may be difficult to realize on timescales comparable to the disk lifetime (Myr), necessitating periodic deposition of mass from the reservoir onto the star, and making this scenario similar to the aforementioned instability in the inner disk.

Alternatively, gas reaching the inner regions of the disk may be lost in a wind (photoevaporative or MHD driven). It can also be consumed by vigorously accreting giant planets residing in the inner disk. If that were the case, then in many systems the rate of mass removal from the global inward accretion flow should be matching the accretion rate set on large scales, with only a small amount of mass reaching the star. The wind is likely to also affect the angular momentum budget of the disk in a nontrivial manner, a possibility that we consider next.

\subsection{Nonviscous Evolution of the Protoplanetary Disks}

One final, very intriguing possibility is that the angular momentum and mass transport in the protoplanetary disks have a nonviscous (nondiffusive) character. In this case Equations (1)-(3) do not hold, and an $\alpha-\dot{M}$ correlation 
emerges simply as a consequence of calculating $\alpha$ through Equation (2), with no real physical meaning for $\alpha$. Also, stellar $\dot{M}$ may have little to do with the global disk parameters, although the work of Manara et al. (2016) does show evidence for a correlation between $M_{d}$ and $\dot{M}$ (which is not obvious in our sample of resolved disks).

Such nonviscous transport may be caused by the magnetically controlled winds (Blandford \& Payne 1982; Konigl 1989; Wang \& Goodman 2017). Outflows from the disks of YSOs are a well-studied observational phenomenon (Frank et al. 2014). Recently self-consistent launching of the magnetocentrifugal winds has been observed in simulations of magnetized accretion disks (Suzuki \& Inutsuka 2009; Bai \& Stone 2013), adding support to this possibility.

Another potential driver of the nondiffusive evolution of the protoplanetary disks could be the density waves excited by massive perturbers, e.g., planets or stellar companions (Goodman \& Rafikov 2001; Rafikov 2002, 2016; Dong et al. 2016). Global spiral waves have been observed recently in several protoplanetary disks, e.g., in SAO 206462 (Garufi et al. 2013), MWC 758 (Benisty et al. 2015), HD 100453 (Wagner et al. 2015), etc.

We believe that in light of the perceived difficulty of the known local turbulent transport mechanisms to drive the protoplanetary disk evolution on megayear timescales (Turner et al. 2014), the nondiffusive mechanisms for driving disk evolution should be considered very seriously. Our work may thus provide strong indirect evidence in favor of this possibility.

\subsection{Comparison with Previous Studies}

There have been a handful of studies trying to understand viscous evolution of the protoplanetary disks based on observational data. Using the mean properties of a large sample of the protoplanetary disks, Hartmann et al. (1998) have concluded that their effective viscosity should be narrowly clustered around $\alpha \approx 10^{-2}$. In this work we determine $\alpha$ for individual objects and find a much broader distribution of $\alpha$, extending over more than two orders of magnitude (Figure 2). This difference suggests that care should be taken when making inferences based on the averaged properties of the sample.

Some studies of viscous evolution of the protoplanetary disks have tried to verify Equation (3) with $t_{\nu}$ set equal to the age of the system $t_{\star}$ (Hartmann et al. 1998; Jones et al. 2012; Manara et al. 2016). Identification of $t_{\nu}$ with the age of the central star is a valid procedure as long as $t_{\star}$ exceeds the viscous time of the disk at its initial radius $r_{0}$. This assumption is similar to our implicit assumption that the current disk size exceeds its initial size, $r_{\text {out }} \gtrsim r_{0}$, so that viscous evolution enters the self-similar regime and the memory of initial conditions gets erased. However, the problem with using this metric of disk evolution is that the determination of ages of the young stars is notoriously difficult (Soderblom et al. 2014)

Despite this drawback, Hartmann et al. (1998) and Jones et al. (2012) have tried to verify that $M_{d} / \dot{M} \sim t_{\star}$ (up to a constant factor of order unity) using observational data. Both studies found significant deviations (up to two orders of magnitude) from this simple relation. In Figure 12 we show the characteristic accretion time for the objects in our sample, plotted against $\dot{M}$ and $M_{d}$. Dotted lines show the range of ages for the Lupus objects in our sample (Alcalá et al. 2016; we do

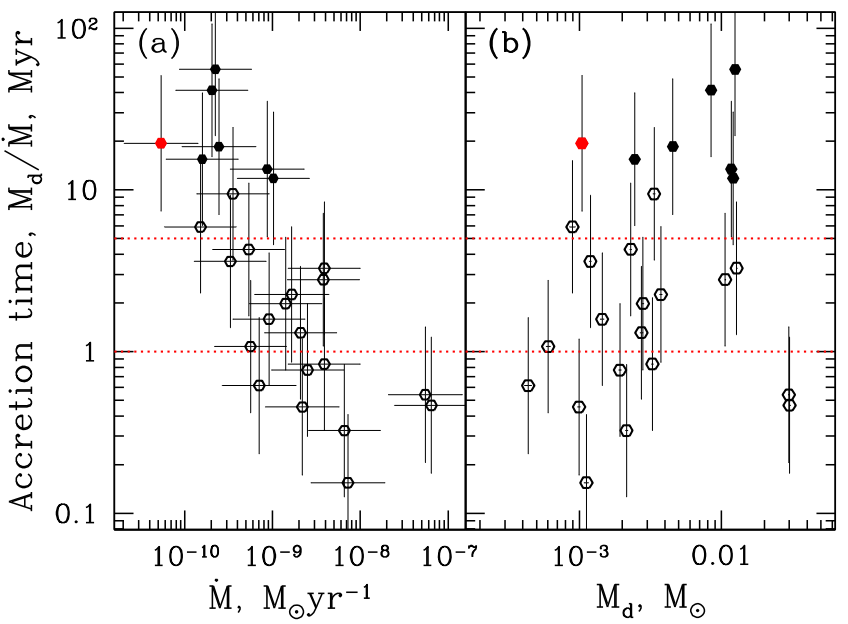

Figure 12. Characteristic accretion time $M_{d} / \dot{M}$ shown as a function of (a) $\dot{M}$ and (b) $M_{d}$. Dotted lines illustrate the approximate upper and lower age limits for the objects in Lupus (Alcalá et al. 2014).

not attempt to use uncertain ages of the individual objects). It is clear that accretion times of many objects fall outside this range, by more than an order of magnitude in some cases. This agrees with the conclusions of Hartmann et al. (1998) and Jones et al. (2012).

In Figure 12(a) one can also see a strong anticorrelation between $M_{d} / \dot{M}$ and $\dot{M}$, with a much weaker statistical connection for $M_{d}$ (see Figure 12(b)). This demonstrates the key role of $\dot{M}$ for the accretion time, just as we found for $\alpha$ in Section 3.2.

Jones et al. (2012) were not able to account for the discrepancy between $M_{d} / \dot{M}$ and $t_{\star}$ even using sophisticated disk models including the effects of dead zones, photoevaporation, planet formation, etc. Instead, they concluded that it follows from the systematic errors in the determination of $M_{d}$. However, we find that the systematic underestimate (or overestimate) of $M_{d}$ would result in a uniform overestimate (underestimate) of $\alpha$, but would not explain the emergence of the $\alpha-\dot{M}$ correlation. Our results suggest that the discrepancy between $M_{d} / \dot{M}$ and $t_{\star}$ is more likely to be caused by the decoupling of the central $\dot{M}$ from the global mass accretion rate $M_{d} / t_{\nu}$ computed using the standard theory of viscous disk evolution, as described in Sections 4.4-4.5.

We also note that the scenario in which $\alpha$ is controlled by a yet-unidentified variable (Section 4.3) is not expected to produce significant deviations from the $M_{d} / \dot{M} \sim t_{\star}$ relation (which is insensitive to $\alpha$ in the self-similar regime). This may argue against this scenario for the protoplanetary disks, although more work is certainly needed to resolve this issue.

Our approach bypasses the issue of uncertain stellar ages by simply ignoring them altogether. Instead, we use spatial information to gain insight on the physical mechanisms responsible for the angular momentum transport in the protoplanetary disks by measuring $\alpha$. Past efforts (Hartmann et al. 1998; Jones et al. 2012) did not have the ability to do that because they lacked accurate information on the sizes of disks in individual objects. Thus, our work represents an independent way of testing the theory of viscous evolution of the protoplanetary disks. 


\subsection{Implications for the $\dot{M}-M_{\star}$ Correlation}

It has long been known that accretion rates onto young stars tend to scale rather strongly with the stellar mass $M_{\star}$, albeit with a large scatter (Muzerolle et al. 2003; Alcalá et al. 2016). To explain this correlation, several models were proposed (Alexander \& Armitage 2006; Dullemond et al. 2006), suggesting that the $\dot{M}-M_{\star}$ relation reflects the dependence of initial conditions for the disk evolution on the stellar mass. The substantial scatter around the $\dot{M}-M_{\star}$ relation has been explained as arising as a result of the spread in ages of objects (Alexander \& Armitage 2006) or initial angular momentum of their disks (Dullemond et al. 2006). A generic feature of all such models is their reliance on simple viscous models for the subsequent disk evolution, which use a single value of $\alpha$ (independent of radius, for all systems) to predict the central accretion rate $\dot{M}$. Our results strongly suggest that such an interpretation may be problematic.

Even if the disk evolution is truly viscous and can be characterized by a single (global) value of $\alpha$ in a given system, as assumed by Alexander \& Armitage (2006) and Dullemond et al. (2006), our interpretation of the $\alpha-\dot{M}$ correlation presented in Section 4.3 still implies a significant variation of $\alpha$ (by orders of magnitude) between different objects. Whether the $\dot{M}-M_{\star}$ correlation would hold once the variation of $\alpha$ (dependent on yet-undetermined disk characteristics) is accounted for in these viscous models is not clear at the moment.

The relevance of viscous models for explaining the $\dot{M}-M_{\star}$ correlation is even less clear if stellar $\dot{M}$ is decoupled from the global value of the accretion rate, as discussed in Sections 4.4 and 4.5. Viscous models necessarily assume that the global disk properties uniquely determine stellar $\dot{M}$, which may not be the case, as we have shown. On the other hand, one could argue that the decoupling of stellar $\dot{M}$ from the global accretion rate is the ultimate cause of the large scatter in the $\dot{M}-M_{\star}$ relation (more so than the dispersion of stellar ages or initial conditions for the disk evolution).

In any case, our results strongly suggest that any interpretation of the $\dot{M}-M_{\star}$ correlation should be based on a clear understanding of what physics is actually reflected in the observed stellar accretion rates.

\section{Summary}

In this work we explored viscous evolution of the protoplanetary disks. Using an observational sample of 26 disks resolved with ALMA with measured masses (based on submillimeter continuum) and central accretion rates, we derived the values of the dimensionless viscosity parameter $\alpha$, with the goal of constraining the mechanism of the angular momentum transport in the disk. Our findings can be summarized as follows.

1. The distribution of inferred values of $\alpha$ extends over more than two orders of magnitude, from $10^{-4}$ to 0.04 , with no obvious preferred value inside this interval.

2. We found no correlation of $\alpha$ with either the global disk parameters (mass, size, surface density) or stellar parameters (luminosity, mass, radius).

3. The main finding of this work is the discovery of a strong linear correlation between $\alpha$ and central mass accretion rate $\dot{M}$, which is robust with regard to the thermodynamic assumptions about the disk. This correlation persists even if we use the CO-based gas masses for computing $\alpha$, and holds not only for $\alpha$ but also for the dimensional kinematic viscosity $\nu$ on global scales.

These results suggest that a simple picture in which viscous evolution of the protoplanetary disks is driven by a physical process (e.g., MRI) with a single, well-defined value of $\alpha$ is too simplistic and must be modified. We find that observational errors and biases cannot account for the observed $\alpha-\dot{M}$ correlation, and we seek other explanations. We find it unlikely that gas accretion onto the stellar surface can have a direct effect on $\alpha$ (e.g., through the accretional energy release) on scales of order the disk size (tens to hundreds of au). We propose three other possibilities for explaining the $\alpha-\dot{M}$ correlation, which effectively assume that either $\alpha$ or $\dot{M}$ is decoupled from the global characteristics (mass, size) of the disk. In that case Equation (2) naturally leads to a linear relation between $\alpha$ and $\dot{M}$. These possibilities are as follows.

1. The value of $\alpha$ in every disk is controlled by some yetunobserved variable, variation of which is responsible for the broad range of $\alpha$. This, in turn, is the main cause of the variation of $\dot{M}$. In the case of accretion driven by the (nonideal) MRI, the role of such a control parameter may be played by the disk ionization, as well as the strength or geometry of the magnetic field in the disk.

2. Stellar $\dot{M}$ may be decoupled from the global mass accretion rate by some instability operating in the inner disk, or mass accumulation in a dead zone, or a wind with high mass-loss rate. In this case the inferred values of $\alpha$ do not characterize the global disk evolution.

3. Finally, disk evolution may have a nondiffusive (nonviscous) character, in which case the inferred $\alpha$ has no physical meaning. This may be the case if mass accretion in protoplanetary disks is driven by, e.g., magnetocentrifugal winds or spiral density waves.

Future work aimed at expanding the sample of resolved protoplanetary disks with well-measured masses and accretion rates will help us identify the physical reason behind the observed $\alpha-\dot{M}$ correlation.

I am indebted to Megan Ansdell and Juan Manuel Alcalá for sharing their data with me, to Carlo Felice Manara and Eugene Churazov for useful discussions, to Ruobing Dong for insightful comments on the manuscript, and to an anonymous referee for the constructive and detailed review. Financial support for this study has been provided by the NSF via grant AST-1409524 and NASA via grant 15-XRP15-2-0139.

\section{References}

Alcalá, J. M., Manara, C. F., Natta, A., et al. 2016, arXiv:1612.07054

Alcalá, J. M., Natta, A., Manara, C. F., et al. 2014, A\&A, 561, A2 Alexander, R. D., \& Armitage, P. J. 2006, ApJL, 639, L83

Andrews, S. M., Wilner, D. J., Hughes, A. M., et al. 2012, ApJ, 744, 162 Ansdell, M., Williams, J. P., van der Marel, N., et al. 2016, ApJ, 828, 46 Audard, M., Ábrahám, P., Dunham, M. M., et al. 2014, in Protostars and

Planets VI, ed. H. Beuther et al. (Tucson, AZ: Univ. Arizona Press), 387 Bai, X.-N., \& Stone, J. M. 2011, ApJ, 736, 144

Bai, X.-N., \& Stone, J. M. 2013, ApJ, 769, 76 Balbus, S. A., \& Hawley, J. F. 1991, ApJ, 376, 214

Benisty, M., Juhasz, A., Boccaletti, A., et al. 2015, A\&A, 578, L6

Birnstiel, T., \& Andrews, S. M. 2014, ApJ, 780, 153

Blandford, R. D., \& Payne, D. G. 1982, MNRAS, 199, 883

Calvet, N., \& Gullbring, E. 1998, ApJ, 509, 802 
Calvet, N., Hartmann, L., \& Strom, S. E. 2000, in Protostars and Planets IV, ed. V. Manning, A. P. Boss, \& S. S. Russell (Tucson, AZ: Univ. Arizona Press), 377

Chandrasekhar, S. 1960, PNAS, 46, 253

Chiang, E. I., \& Goldreich, P. 1997, ApJ, 490, 368

Cleeves, L. I., Öberg, K. I., Wilner, D. J., et al. 2016, ApJ, 832, 110

Dong, R., Zhu, Z., Fung, J., et al. 2016, ApJL, 816, L12

Dullemond, C. P., Natta, A., \& Testi, L. 2006, ApJL, 645, L69

Fleming, T. P., Stone, J. M., \& Hawley, J. F. 2000, ApJ, 530, 464

Frank, A., Ray, T. P., Cabrit, S., et al. 2014, in Protostars and Planets VI, ed. H. Beuther et al. (Tucson, AZ: Univ. Arizona Press), 451

Gammie, C. F. 1996, ApJ, 457, 355

Gammie, C. F. 2001, ApJ, 553, 174

Garufi, A., Quanz, S. P., Avenhaus, H., et al. 2013, A\&A, 560, A105

Glassgold, A. E., Feigelson, E. D., \& Montmerle, T. 2000, in Protostars and

Planets IV, ed. V. Manning, A. P. Boss, \& S. S. Russell (Tucson, AZ: Univ. Arizona Press), 429

Goodman, J., \& Rafikov, R. R. 2001, ApJ, 552, 793

Hartmann, L., Calvet, N., Gullbring, E., \& D’Alessio, P. 1998, ApJ, 495, 385

Hillenbrand, L. A. 2005, arXiv:astro-ph/0511083

Isobe, T., Feigelson, E. D., Akritas, M. G., \& Babu, G. J. 1990, ApJ, 364, 104

Jin, L. 1996, ApJ, 457, 798

Jones, M. G., Pringle, J. E., \& Alexander, R. D. 2012, MNRAS, 419, 925

Konigl, A. 1989, ApJ, 342, 208

Latter, H. N. 2016, MNRAS, 455, 2608

Lin, M.-K., \& Youdin, A. N. 2015, ApJ, 811, 17

Lovelace, R. V. E., Li, H., Colgate, S. A., \& Nelson, A. F. 1999, ApJ, 513, 805

Lynden-Bell, D., \& Pringle, J. E. 1974, MNRAS, 168, 603

Manara, C. F., Rosotti, G., Testi, L., et al. 2016, A\&A, 591, L3
Miotello, A., van Dishoeck, E. F., Kama, M., \& Bruderer, S. 2016a, A\&A, 594, A85

Miotello, A., van Dishoeck, E. F., Williams, J. P., et al. 2016b, arXiv:1612.01538

Muzerolle, J., Hillenbrand, L., Calvet, N., Briceño, C., \& Hartmann, L. 2003, ApJ, 592, 266

Pascucci, I., Testi, L., Herczeg, G. J., et al. 2016, ApJ, 831, 125

Rafikov, R. R. 2002, ApJ, 569, 997

Rafikov, R. R. 2015, ApJ, 804, 62

Rafikov, R. R. 2016, ApJ, 831, 122

Ross, J., Latter, H. N., \& Guilet, J. 2016, MNRAS, 455, 526

Schwarz, K. R., Bergin, E. A., Cleeves, L. I., et al. 2016, ApJ, 823, 91

Shakura, N. I., \& Sunyaev, R. A. 1973, A\&A, 24, 337

Simon, J. B., Bai, X.-N., Armitage, P. J., Stone, J. M., \& Beckwith, K. 2013a, ApJ, 775, 73

Simon, J. B., Bai, X.-N., Stone, J. M., Armitage, P. J., \& Beckwith, K. 2013b, ApJ, 764, 66

Soderblom, D. R., Hillenbrand, L. A., Jeffries, R. D., Mamajek, E. E., \& Naylor, T. 2014, in Protostars and Planets VI, ed. H. Beuther et al. (Tucson, AZ: Univ. Arizona Press), 219

Stoll, M. H. R., \& Kley, W. 2014, A\&A, 572, A77

Suzuki, T. K., \& Inutsuka, S.-i 2009, ApJL, 691, L49

Turner, N. J., Fromang, S., Gammie, C., et al. 2014, in Protostars and Planets VI, ed. H. Beuther et al. (Tucson, AZ: Univ. Arizona Press), 411

Urpin, V., \& Brandenburg, A. 1998, MNRAS, 294, 399

Velikhov, E. P. 1959, JETP, 36, 1398

Wagner, K., Apai, D., Kasper, M., \& Robberto, M. 2015, ApJL, 813, L2

Walsh, C., Juhász, A., Meeus, G., et al. 2016, ApJ, 831, 200

Wang, L., \& Goodman, J. J. 2017, ApJ, 835, 59

Wardle, M., \& Koenigl, A. 1993, ApJ, 410, 218 